\title{
Subsurface Oceans on Europa and Callisto: Constraints from Galileo Magnetometer Observations
}

\author{
Christophe Zimmer and Krishan K. Khurana \\ Institute of Geophysics and Planetary Physics, University of California—Los Angeles, Los Angeles, California 90095-1567 \\ E-mail: czimmer@igpp.ucla.edu
}

and

Margaret G. Kivelson

Institute of Geophysics and Planetary Physics, and Department of Earth and Space Sciences, University of California-Los Angeles, Los Angeles, California 90095-1567

Received September 22, 1999; revised May 31, 2000

\begin{abstract}
Magnetic field perturbations measured during Galileo flybys of E uropa and Callisto are consistent with dipole fields induced by the temporal variations of the ambient jovian magnetospheric field. These fields are close to those expected for perfectly conducting moons. We investigate the implications of these observations for the electrical structure of the moon's interiors using a simple shell model. It is found that E uropa and Callisto must possess regions where the conductivity exceeds 0.06 and $0.02 \mathrm{~S} / \mathrm{m}$ at a depth of less than 200 and $300 \mathrm{~km}$ below the surface, respectively. This conductivity is unattainable in ice or silicates, unless the ice layer is at least partially molten or very large temperature gradients can be maintained below the ice. An ionosphere or a cloud of pick-up ions are probably also insufficiently conductive. G lobal E arth-like oceans under the surface of both moons could account for the observations provided they are at least a few kilometers thick. (c) 2000 Academic Press

Key Words: E uropa; Callisto; interiors; magnetic fields; J upiter, magnetosphere.
\end{abstract}

\section{INTRODUCTION}

Because Jupiter's magnetic dipole axis is tilted with respect to its rotation axis, the Galilean moons orbiting in the jovigraphic equatorial plane experience a magnetic field that varies periodically at the apparent rotation frequency of Jupiter, i.e., the synodic frequency. It was first suggested by Colburn and Reynolds (1985) that this time-varying field could induce electric currents inside the moons, provided that regions of sufficient electrical conductivity exist in their interiors. These currents would produce a secondary (or induced) magnetic field that adds to the background field of Jupiter and to fields arising from currents flowing in the ambient plasma to give the total observed field. Khurana et al. (1998) and Kivelson et al. (1999) recently showed that the dominant large-scale features of magnetic field perturbations observed by the Galileo spacecraft during flybys of both Europa and Callisto are consistent with induced magnetic dipoles, as had also been pointed out by Neubauer (1998). At both moons, the observed magnetic field perturbations are approximately those expected for moons responding as perfectly conducting spheres. Such a response requires a globally distributed highly conducting medium located close to the surface of the satellites. Khurana et al. (1998) and Kivelson et al. (1999) interpreted this result as support for the presence of salty subsurface oceans. For Europa, the same conclusion was reached independently by K. Kuramoto et al. (unpublished manuscript, 1998), extending a previous study by Kuramoto et al. (1998).

In this paper, we seek to further constrain the nature and depth of the conducting mediums of Europa and Callisto on the basis of Galileo magnetometer observations. In Section 2, we discuss properties of the primary (or inducing) magnetic field important for this study. In Section 3, we compute the field induced by a uniform spherical shell, chosen as a simple model of the electrical structure of the moon. In Section 4, we examine Galileo observations at Europa and Callisto to characterize the induced dipole moments. In Section 5 we combine the results of Sections 3 and 4 to constrain the conductivity and spatial dimensions of the hypothesized subsurface oceans. In Section 6 we examine alternative candidates for the high-conductivity regions. In Section 7 we summarize and discuss our results.

\section{THE PRIMARY FIELD}

We begin with a description of some features of the primary field that are of interest in the analysis of the following sections. Jupiter's moons experience a time-varying field which can be divided into the large-scale magnetospheric background field and the field generated by local plasma currents caused by the nonelectromagnetic interaction with the moon. In most of this 
paper (except in Sections 4.2.1 and 6.2), we ignore the effect of local plasma currents. The inducing field then arises only from the background field, which consists of the internal field of Jupiter and the contribution from large-scale magnetospheric current systems. This field varies on a spatial scale of at least $1 R_{\mathrm{J}}$ (= radius of Jupiter $=71492 \mathrm{~km}$ ) and can thus be considered uniform on the spatial scale of the moons $\left(R_{\mathrm{E}}=\right.$ radius of Europa $=1560 \mathrm{~km} ; R_{\mathrm{C}}=$ radius of Callisto $\left.=2409 \mathrm{~km}\right)$.

Figures 1a and 1b of Kivelson et al. (1999) each show one cycle of Jupiter's background field at the location of Europa and Callisto respectively, as a function of the moon's west longitude, or equivalently as a function of time during one synodic period (11.23 $\mathrm{h}$ for Europa and $10.18 \mathrm{~h}$ for Callisto). The field was computed from the empirical model of Khurana (1997), which includes the contribution of the current sheet and the $\mathrm{O}_{6}$ model of Connerney (1992) for Jupiter's internal field. The radial field component $\left(B_{\mathrm{r}}\right)$ oscillates with an amplitude of 200-250 nT at Europa, and $40 \mathrm{nT}$ at Callisto. The azimuthal component $\left(B_{\varphi}\right)$ oscillates with an amplitude of 60-75 nT at Europa, and $8 \mathrm{nT}$ at Callisto. As illustrated in Fig. 1 of Khurana et al. (1998), the equatorial field projection is roughly elliptically polarized at Europa, where the field is mainly determined by Jupiter's tilted dipole moment, and almost linearly polarized at Callisto, where the field is largely controlled by azimuthal currents flowing in the thin plasma sheet. At both moons, the north-south component of the field $\left(B_{\theta}\right)$ remains essentially constant (i.e., its amplitude of oscillation is small compared to that of $B_{\mathrm{r}}$ and $B_{\varphi}$ ). The constancy of $B_{\theta}$ is due to the fact that near the equatorial plane of Jupiter (i) the contribution to $B_{\theta}$ from the tilted dipole field depends mainly on the radial distance from the dipole center, which is approximately constant along the moon's orbit, and (ii) the contribution to $B_{\theta}$ from the thin current sheet varies much less across the current sheet than the equatorial field components (Vasyliunas 1983).

Because of the strong latitudinal confinement of the current sheet, Callisto spends most of its time in the lobes, where both $B_{\mathrm{r}}$ and $B_{\varphi}$ are almost uniform. As a result, this moon is exposed to a roughly constant field during most of the time interval between consecutive current sheet crossings (see Fig. 1b of Kivelson et al. 1999). This is not the case of Europa, where the importance of the current sheet field relative to Jupiter's dipole field is small.

It can also be noted from Figs. 1a of 1b of Kivelson et al. (1999) that the average of $B_{\mathrm{r}}$ and $B_{\varphi}$ over one synodic cycle are both close to 0 (i.e., small compared to their oscillation amplitudes). Together with the near-constancy of $B_{\theta}$, this implies that the inducing field, which is the time-varying component of the external field seen by the moon, is simply given by the equatorial projection of Jupiter's background field. This property will be used to model the induced field signature in Section 4.

Throughout this paper, we will assume that the primary field varies solely at the synodic frequency. In reality, the primary field is expected to exhibit variations at several other frequencies, notably the orbital frequency of the moons (as a result of the eccentricity and inclination of the moon's orbit), harmonics of the synodic period (because of the nonsinusoidal character of the field variations induced by the current sheet, as apparent in Figs. 1a and 1b of Kivelson et al. 1999), and related beat frequencies. However, estimates of the power associated with those frequencies using Khurana's (1997) model of the jovian field show that for both moons the synodic variation dominates by roughly an order of magnitude, so that other frequencies can be ignored in a first-order treatment.

\section{MAGNETIC FIELD INDUCED BY A UNIFORMLY CONDUCTING SHELL}

As a simple representation of the electrical structure of the moon, we adopt the model illustrated in Fig. 1 of a shell of uniform conductivity $\sigma$, inner radius $r_{1}$, and outer radius $r_{0}=$ $r_{1}+h$, surrounding an insulating core and surrounded by an insulating shell of inner radius $r_{0}$ and outer radius $r_{\mathrm{m}}=r_{0}+d$, where $r_{\mathrm{m}}$ is the radius of the moon. This is the simplest model that allows us to constrain the conductivity $(\sigma)$, thickness $(h)$, and depth $(d)$ of a conducting layer such as a salty ocean.

The response of a uniformly conducting spherical shell to a uniform time-varying magnetic field is a classical problem of electromagnetic theory. In the following we only recall the main assumptions and results and refer to Parkinson (1983) for details of the derivation.

Inside the conducting shell, the combination of Maxwell's equations and Ohm's law yields the diffusion equation for the magnetic field

$$
\nabla^{2} \mathbf{B}=\mu \sigma \frac{\partial \mathbf{B}}{\partial t},
$$

where $\mu$ is the permeability, taken to be everywhere equal to the vacuum permeability $\mu_{0}$. In the insulating regions of the moon,

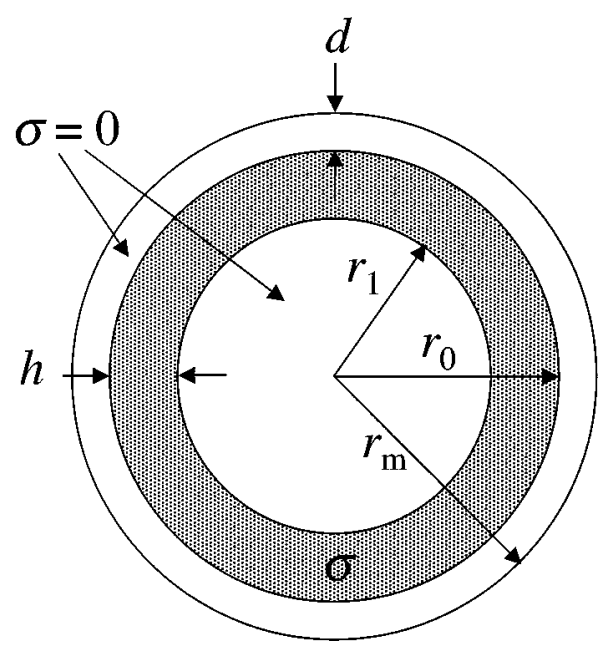

FIG . 1. The assumed conductivity model: a shell of uniform conductivity $\sigma$ surrounded by an insulating layer and surrounding an insulating core. 
the field satisfies

$$
\nabla^{2} \mathbf{B}=0
$$

The same equation holds outside the moon where the conductivity is small, provided that plasma convection effects are neglected (flow velocity $\mathbf{v}=0$ ). The displacement current was ignored to obtain Eqs. (1) and (2).

We now assume that the primary magnetic field oscillates at the frequency $\omega$ along a specified direction with unit vector $\mathbf{e}_{0}$ and write it as the real part of the complex vector $\mathbf{B}_{\text {prim }}=$ $B_{\text {prim }} e^{-i \omega t} \mathbf{e}_{0}$. The actual time-varying field does not necessarily oscillate at a single frequency or in a single direction, but this field and the corresponding solutions of the (linear) equations (1) and (2) can always be represented as a linear superposition of monochromatic and linearly polarized fields. The boundary conditions to be satisfied by the total time-varying field $\mathbf{B}$ are:

(i) B must be continuous across the boundaries of each shell (the normal component of the field is continuous and the tangential component is continuous because the permeability $\mu$ is uniform);

(ii) $\mathbf{B}$ must not be infinite at the center of the body, $\mathbf{r}=0$; and

(iii) $\mathbf{B}$ must be asymptotically equal to the external field $\mathbf{B}_{\text {prim }}$ far away from the body $\left(r=|\mathbf{r}| \gg r_{0}\right)$.

The solution field can be written $\mathbf{B}=\mathbf{B}_{\text {prim }}+\mathbf{B}_{\text {sec }}$, where $\mathbf{B}_{\text {sec }}$ is the secondary field. For the simple shell model considered here, an analytical expression can be found for $\mathbf{B}$. Because the primary field is assumed uniform (see Section 2) and the conductivity distribution has spherical symmetry, the induced field outside the conductor $\left(r>r_{0}\right)$ is a dipole field

$$
\mathbf{B}_{\mathrm{sec}}=\frac{\mu_{0}}{4 \pi}\left[3(\mathbf{r} \cdot \mathbf{M}) \mathbf{r}-r^{2} \mathbf{M}\right] / r^{5},
$$

whose moment $\mathbf{M}$ oscillates at the same frequency $\omega$ and along the same direction $\mathbf{e}_{0}$ as the primary field. The moment can therefore be written

$$
\mathbf{M}=-\frac{4 \pi}{\mu_{0}} A e^{i \phi} \mathbf{B}_{\text {prim }} r_{\mathrm{m}}^{3} / 2
$$

so that Eq. (3) becomes

$$
\mathbf{B}_{\mathrm{sec}}=-A e^{-i(\omega t-\phi)} B_{\text {prim }}\left[3\left(\mathbf{r} \cdot \mathbf{e}_{0}\right) \mathbf{r}-r^{2} \mathbf{e}_{0}\right] r_{\mathrm{m}}^{2} /\left(2 r^{5}\right) .
$$

The parameters $A$ and $\phi$ are real numbers, which after Parkinson (1983) are given by the complex equations

$$
\begin{aligned}
A e^{i \phi} & =\left(\frac{r_{0}}{r_{\mathrm{m}}}\right)^{3} \frac{R J_{5 / 2}\left(r_{0} k\right)-J_{-5 / 2}\left(r_{0} k\right)}{R J_{1 / 2}\left(r_{0} k\right)-J_{-1 / 2}\left(r_{0} k\right)} \\
R & =\frac{r_{1} k J_{-5 / 2}\left(r_{1} k\right)}{3 J_{3 / 2}\left(r_{1} k\right)-r_{1} k J_{1 / 2}\left(r_{1} k\right)},
\end{aligned}
$$

where $k=(1-i) \sqrt{\mu_{0} \sigma \omega / 2}$ has the dimension of a (complex) wave vector, $J_{m}$ is the Bessel function of first kind and order $m$ (expressions are given in the Appendix), and the radii $r_{0}, r_{1}$, and $r_{\mathrm{m}}$ are defined as above (see Fig. 1). The physical induced field is given by the real part of Eq. (4). The scalars $A$ and $\phi$ are thus the (normalized) amplitude and the phase lag, respectively, of the induced dipole moment relative to the primary field. Over one oscillation period $2 \pi / \omega$, the equatorial induced field at the surface of the moon $\left(\mathbf{r} \cdot \mathbf{e}_{0}=\mathbf{0}, r=r_{\mathrm{m}}\right)$ reaches a maximum intensity $B_{\text {sec,eq }}=A B_{\text {prim }} / 2$. Equations (5) and (6) show that both $A$ and $\phi$ depend only on the three nondimensional parameters $r_{0} / r_{\mathrm{m}}, r_{0} k$, and $r_{1} k$, or equivalently on the numbers $r_{0} / r_{\mathrm{m}}, r_{0} / s$, and $r_{1} / s$, where $s=\left(\mu_{0} \sigma \omega / 2\right)^{-1 / 2}$. The latter quantity, $s$, is the skin depth, which is the $e$-folding penetration distance of the time-varying field into a semi-infinite plane conductor of conductivity $\sigma$. Alternatively, $A$ and $\phi$ can be expressed as functions of the three nondimensional parameters $d / r_{\mathrm{m}}, h / r_{\mathrm{m}}$, and $\sigma / \sigma_{\mathrm{m}}$, where we introduced a reference conductivity $\sigma_{\mathrm{m}}=2 /\left(\mu_{0} \omega r_{\mathrm{m}}^{2}\right)$, for which the skin depth is equal to the radius of the moon $\left(s=r_{\mathrm{m}}\right)$.

We now examine some properties of the solution, starting with the limit $\sigma / \sigma_{\mathrm{m}} \rightarrow \infty$, which we refer to as the perfect conductor case. In that case, Eqs. (5) and (6) reduce to $A=\left(r_{0} / r_{\mathrm{m}}\right)^{3}$ and $\phi=0$. As can be verified from Eq. (4), $\mathbf{B}_{\text {sec }}$ then exactly cancels out $\mathbf{B}_{\text {prim }}$ at the two points on the outer surface of the conductor where $\mathbf{B}_{\text {prim }}$ is normal to that surface $\left(\mathbf{r}= \pm r_{0} \mathbf{e}_{0}\right)$. The total timevarying field, $\mathbf{B}=\mathbf{B}_{\text {prim }}+\mathbf{B}_{\text {sec }}$, can be seen to be everywhere tangent to the outer surface of the perfect conductor. This is expected because a time-varying field does not penetrate the interior of a perfect conductor. Note however that field lines may still thread a perfectly conducting moon because of the presence of a constant field component, such as $B_{\theta}$ in Section 2, in addition to the time-varying component (this is in contrast to the total exclusion of magnetic fields by a superconductor). In the following we shall denote the induced field for the case of a perfectly conducting moon (i.e., a perfect spherical conductor of radius $r_{\mathrm{m}}$ ) by $\mathbf{B}_{\mathrm{sec}, \infty}$.

For a conducting shell with arbitrary conductivity and size, the secondary field, given by Eq. (4), can be simply related to the field induced by a perfectly conducting moon through $\mathbf{B}_{\mathrm{sec}}=A e^{i \phi} \mathbf{B}_{\mathrm{sec}, \infty}$. For the physical field, i.e., the real part of the previous equation, this translates into

$$
\mathbf{B}_{\mathrm{sec}}(t)=A \mathbf{B}_{\mathrm{sec}, \infty}(t-\phi / \omega) .
$$

Equation (7) states that the instantaneous secondary field produced by a spherical shell of arbitrary conductivity at a time $t$ is equal to the secondary field that would have been induced by a perfectly conducting sphere at an earlier time $t-\phi / \omega$ multiplied by a factor $A$. For $\sigma<\infty$, it can be shown from Eqs. (5) and (6) that the amplitude of the induced field is always smaller than that for a perfect conductor $\left(0 \leq A<\left(r_{0} / r_{\mathrm{m}}\right)^{3}\right)$ and that the induced field always lags behind the primary field $\left(0^{\circ}<\right.$ $\phi \leq 90^{\circ}$ ). The amplitude $A$ vanishes if the shell conductivity 


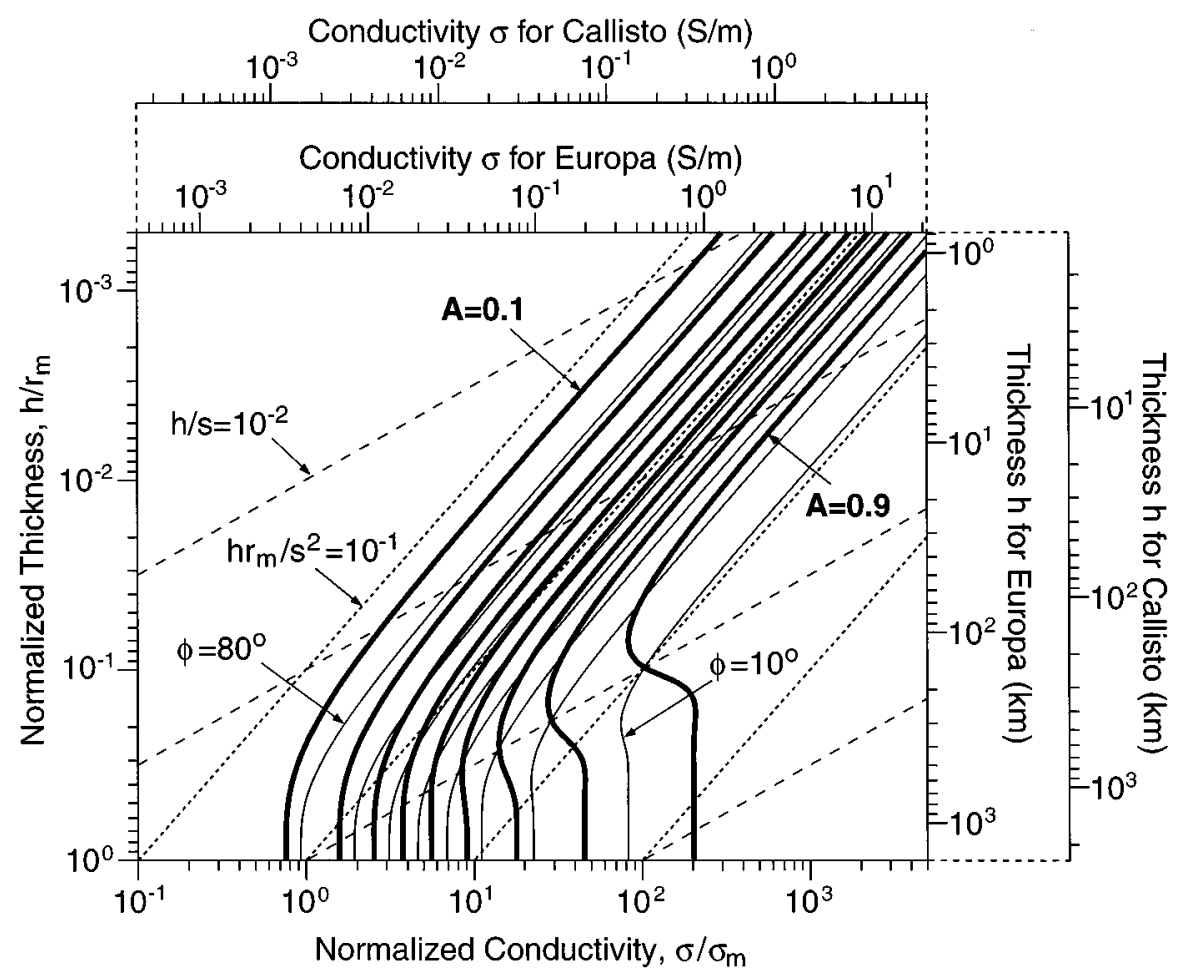

FIG . 2. Normalized amplitude $A$ and phase lag $\phi$ of the induced field as a function of the shell thickness $h$ and the shell conductivity $\sigma$. The thickness $h$ is given in nondimensional units normalized to the moon's radius $r_{\mathrm{m}}$ (equal to the outer radius of the conducting shell), and in kilometers on extra scales appropriate for Europa and Callisto, of radii 1560 and $2409 \mathrm{~km}$, respectively. Similarly, the conductivity $\sigma$ is given in nondimensional units normalized to the reference conductivity $\sigma_{\mathrm{m}}=2 /\left(\mu_{0} \omega r_{\mathrm{m}}^{2}\right)$ and in Sterradians per meter. on scales appropriate for Europa and Callisto, whose reference conductivities are 4.2 and $1.6 \mathrm{mS} / \mathrm{m}$, respectively. The thick solid lines are isocontours of $A$ at $0.1,0.2, \ldots, 0.9$. The thin solid lines are isocontours of $\phi$ at $10^{\circ}, 20^{\circ}, \ldots, 80^{\circ}$. The dotted lines show where $h r_{\mathrm{m}} / s^{2}$ equals $0.1,1,10$, and 100, from left to right. The dashed lines correspond to $h / s=0.01,0.1,1$, and 10 , from left to right.

is low $\left(\sigma \ll \sigma_{\mathrm{m}}\right)$ or if the conductor lies far below the surface $\left(r_{0} \ll r_{\mathrm{m}}\right)$.

We now consider more closely the special case where the shell extends to the surface of the moon, i.e., $d=0$. Figure 2 shows isocontours of $A$ and $\phi$ plotted against $h / r_{\mathrm{m}}$ and $\sigma / \sigma_{\mathrm{m}}$. For a highly conducting shell $\left(\sigma \gg \sigma_{\mathrm{m}}\right)$, the inductive response is close to the perfect conductor limit ( $A \rightarrow 1$ and $\phi \rightarrow 0$ ). For a shell conductivity $\sigma<0.1 \sigma_{\mathrm{m}}$, the amplitude and phase lag are close to those of an insulator: $0 \leq A<0.02$ and $88^{\circ}<\phi \leq 90^{\circ}$. For a fixed shell thickness $h$, the inductive response approaches that of a perfect conductor as the conductivity of the shell increases: the amplitude $A$ increases toward 1 and the phase lag $\phi$ decreases toward 0 . For a fixed value of $\sigma / \sigma_{\mathrm{m}}$, the response initially becomes closer to that of a perfect conductor as the thickness of the shell is increased from 0 . However, if $\sigma / \sigma_{\mathrm{m}}$ is sufficiently large, $A$ and $\phi$ do not vary entirely monotonically with the shell thickness: the largest amplitude and the smallest phase lag are not obtained for a full sphere $\left(h=r_{\mathrm{m}}\right)$, but for some intermediate shell thickness $h_{\max }\left(\sigma / \sigma_{\mathrm{m}}\right), 0<h_{\max }<r_{\mathrm{m}}$. Note also that the shell can be less than a skin depth thick $(h<s)$, yet produce an almost perfect response, provided that the body is large enough. For example, an amplitude $A>0.9$ can be achieved with $h$ smaller than $s / 10$ if $r_{\mathrm{m}}$ is larger than about $30 s$. In fact, a strong inductive response is produced whenever the nondimensional quantity $h r_{\mathrm{m}} / s^{2}=\left(h / r_{\mathrm{m}}\right)\left(\sigma / \sigma_{\mathrm{m}}\right)$ is significantly larger than unity, as is apparent from the dotted lines in Fig. 2. For shells that are thin compared to the body size $\left(h / r_{\mathrm{m}} \ll 1\right)$, an asymptotic expansion of Eqs. (5) and (6) yields $A \approx 2 r_{\mathrm{m}} h / 3 s^{2}$.

For the more general case where $r_{0} \neq r_{\mathrm{m}}$, the phase lag $\phi$ can also be obtained from the thin contours of Fig. 2, but $r_{\mathrm{m}}$ must be replaced by $r_{0}$, and $\sigma_{\mathrm{m}}$ replaced by $\sigma_{0}=2 /\left(\mu_{0} \omega r_{0}^{2}\right)$. With the same substitutions, the thick contours of Fig. 2 indicate $A_{0}$, the amplitude of the induced dipole moment normalized to the dipole moment that would be induced in a perfect conductor of radius $r_{0}$. The normalized amplitude $A$, as defined by Eq. (4), i.e., the ratio of the induced dipole to the dipole induced in a perfect conductor of radius $r_{\mathrm{m}}$, can then be obtained from $A=A_{0}\left(r_{0} / r_{\mathrm{m}}\right)^{3}$.

\section{OBSE RVATIONAL CHARACTERIZATION OF INDUCED FIELDS AT EUROPA AND CALLISTO}

We now turn to the Galileo evidence for induced fields at Europa and Callisto. As stated in the introduction, Khurana et al. (1998) and Kivelson et al. (1999) found a good agreement between the magnetic field perturbations measured by the Galileo magnetometer on different encounters and the perturbations that 
are expected if the moons were perfectly conducting spheres, i.e., if $A=1$ and $\phi=0$. Here we reexamine relevant Galileo observations with the goal of better constraining $A$ and $\phi$. We first show that decreasing $A$ well below unity while maintaining $\phi=0$ produces a poorer fit to the data than a model with $A=1$ (and $\phi=0$ ). Then we address the effect of a finite phase lag $\phi \neq 0^{\circ}$.

\subsection{Induced Field at Callisto}

Prior to October 1999, Galileo encountered Callisto on its 3rd, 9th, 10th, and 20th to 23rd orbits around Jupiter (labeled C3, C9, C10, C20-23). No data were acquired during C20; magnetometer data for $\mathrm{C} 3, \mathrm{C} 9$, and $\mathrm{C} 10$ have been presented earlier by Kivelson et al. (1998). On encounters C10 and C21-23, the magnetic field measured by Galileo agrees poorly with the perturbations expected from induction by a perfectly conducting Callisto. During these flybys, the magnetometer recorded abrupt field fluctuations (with timescales $<5$ min or length scales $<1 R_{\mathrm{C}}$ ) that were larger or comparable in magnitude to the perturbation predicted from induction (see, e.g., Fig. 4c of Kivelson et al. 1999 for C10 data). Because of their strongly localized character, these fluctuations were most probably caused by currents flowing in the ambient plasma, which we do not attempt to model in this study. In this work, we therefore only use data from encounters C3 and C9. The geometry of these Callisto encounters can be read from Fig. 5 of Kivelson et al. (1999). As the primary field was oriented in opposite directions on C3 and C9 (see Fig. 1b of Kivelson et al. 1999 or Fig. 1 of Khurana et al. 1998), an induced dipole moment would have opposite orientations on the two flybys and can thus easily be distinguished from an intrinsic dipole.

4.1.1. Amplitude of the induced field. The thick dots in Figs. $3 \mathrm{a}$ and $3 \mathrm{~b}$ show the magnetic field measured by Galileo during the flybys $\mathrm{C} 3$ and $\mathrm{C} 9$, respectively. We use a right-handed coordinate system that has its origin at the center of the moon, the $x$ axis pointing in the direction of rigid corotation, the $y$ axis pointing toward Jupiter, the $z$ axis parallel to Jupiter's rotation axis, and distances measured in Callisto radii $R_{\mathrm{C}}$. In addition to the measurements are plotted the following fields (computed as explained below): the jovian background field $\mathbf{B}_{\mathrm{J}}$, the total field $\mathbf{B}_{\mathrm{tot}, \infty}$ expected if the moon behaves as a perfect conductor of the same size $(A=1, \phi=0)$, and the total field $\mathbf{B}_{\text {tot }}$ expected for induction with varying values of $A(0.6,0.8,1.35,1.7)$ and a phase lag $\phi=0$.

The background field $\mathbf{B}_{\mathrm{J}}(t)$ was obtained by taking a polynomial fit to the observed field $\mathbf{B}_{\text {obs }}(t)$ during time intervals where the spacecraft was at intermediate distances from the moon, far enough that the perturbation due to the moon could be considered negligible, but close enough to provide a good estimate of the actual background field at the moon's location. This is possible because the background field varies on a spatial scale of order $R_{\mathrm{J}}$ much larger than the typical region of perturbations due to the moon (of order a few $R_{\mathrm{C}}$ ).
From $\mathbf{B}_{\mathrm{J}}$, we estimate the primary field $\mathbf{B}_{\text {prim, }} \mathrm{C} / \mathrm{A}$ at the time of closest approach, $t_{0}$. It is appropriate to ignore the variation of the primary field during the period of the encounter when the induced field signature may be strong, because this time interval (typically less than $30 \mathrm{~min}$, as can be seen from Figs. 3a and 3b) is much shorter than a synodic period. We also make use of the property mentioned earlier (Section 2) that the instantaneous primary field is approximately given by the projection of the background field $\mathbf{B}_{\mathrm{J}}$ on the jovigraphic equatorial plane, so that $B_{\text {prim, } \mathrm{C} / \mathrm{A}, \mathrm{x}}=B_{\mathrm{J}, \mathrm{x}}\left(t_{0}\right), B_{\text {prim, } \mathrm{C} / \mathrm{A}, \mathrm{y}}=B_{\mathrm{J}, \mathrm{y}}\left(t_{0}\right)$, and $B_{\text {prim, } \mathrm{C} / \mathrm{A}, \mathrm{z}}=0$. In the case of a perfectly conducting Callisto, the induced field $\mathbf{B}_{\mathrm{sec}, \infty}(t)$ is then obtained from Eq. (4) by setting $A=1, \phi=0$, $B_{\text {prim }}=\left|\mathbf{B}_{\text {prim,C/A }}\right|, \mathbf{e}_{0}=\mathbf{B}_{\text {prim }, \mathrm{C} / \mathrm{A}} / B_{\text {primeC } / \mathrm{A}}, r_{\mathrm{m}}=R_{C}$, and identifying $\mathbf{r}$ as the time-varying spacecraft position vector measured from the moon's center. The total field for that case, $\mathbf{B}_{\mathrm{tot}, \infty}(t)$, is simply the sum of $\mathbf{B}_{\mathrm{J}}(t)$ and $\mathbf{B}_{\mathrm{sec}, \infty}(t)$. For arbitrary $A$ and $\phi=0^{\circ}$, the total field $\mathbf{B}_{\mathrm{tot}}(t)$ is the sum of $B_{\mathrm{J}}(t)$ and $A \mathbf{B}_{\mathrm{sec}, \infty}(t)$ (see Eq. 7).

As already stated, the measurements are in good agreement with the signature predicted for a perfectly conducting moon ( $A=1, \phi=0)$. They cannot be explained by a stable intrinsic dipole field, since the required dipole moment would need to have switched its orientation between the two encounters. We now seek to refine the estimate of the induced dipole by letting the response factor $A$ differ from 1 and comparing the resulting prediction to the observed field. It is clear from Figs. 3a and $3 \mathrm{~b}$ that the observations are best reproduced with a parameter $A$ close to 1 , say in the range of 0.8 to 1.35 . Values of $A$ outside of this range lead to markedly poorer fits to the data than $A=1$. On the other hand no value of $A$ within this range appears to produce a globally better fit than $A=1$. (Locally, the agreement with the data can be improved with $A \neq 1$, as for example on C9 at the time of closest approach by taking $A=1.35$. However, the $A=1.35$ model is worse at other times, e.g., before 13:44 UT). Because of perturbations due to currents flowing in the plasma and uncertainties in the determination of the background field, it is difficult to constrain the parameter $A$ to a higher level of accuracy. However we believe that an inductive response with $\phi=0$ and $A<0.8$ or $>1.35$ would be significantly harder to reconcile with the observations.

Two remarks must be made at this point. The first is that $A$ can be larger than 1 only if the conductor is larger than the moon itself (see Section 3), a possible situation if the moon is surrounded by a highly conducting medium such as an ionosphere or a cloud of pick-up ions. We will argue in Section 6, however, that none of these media can provide sufficient conductivity for $A$ to exceed 1 noticeably. The second point is that an amplitude $A<1$ is strictly speaking inconsistent with our assumed phase lag $\phi=0$ (see Section 3). We therefore address the effect of a finite phase lag in the following section.

4.1.2. Phase lag of the induced field. The above constraints on the amplitude $A$ were obtained assuming $\phi=0$. They cannot be readily generalized to arbitrary $\phi$ because a finite phase lag $(\phi>0)$ can to some extent mask a reduction of the amplitude $A$. 


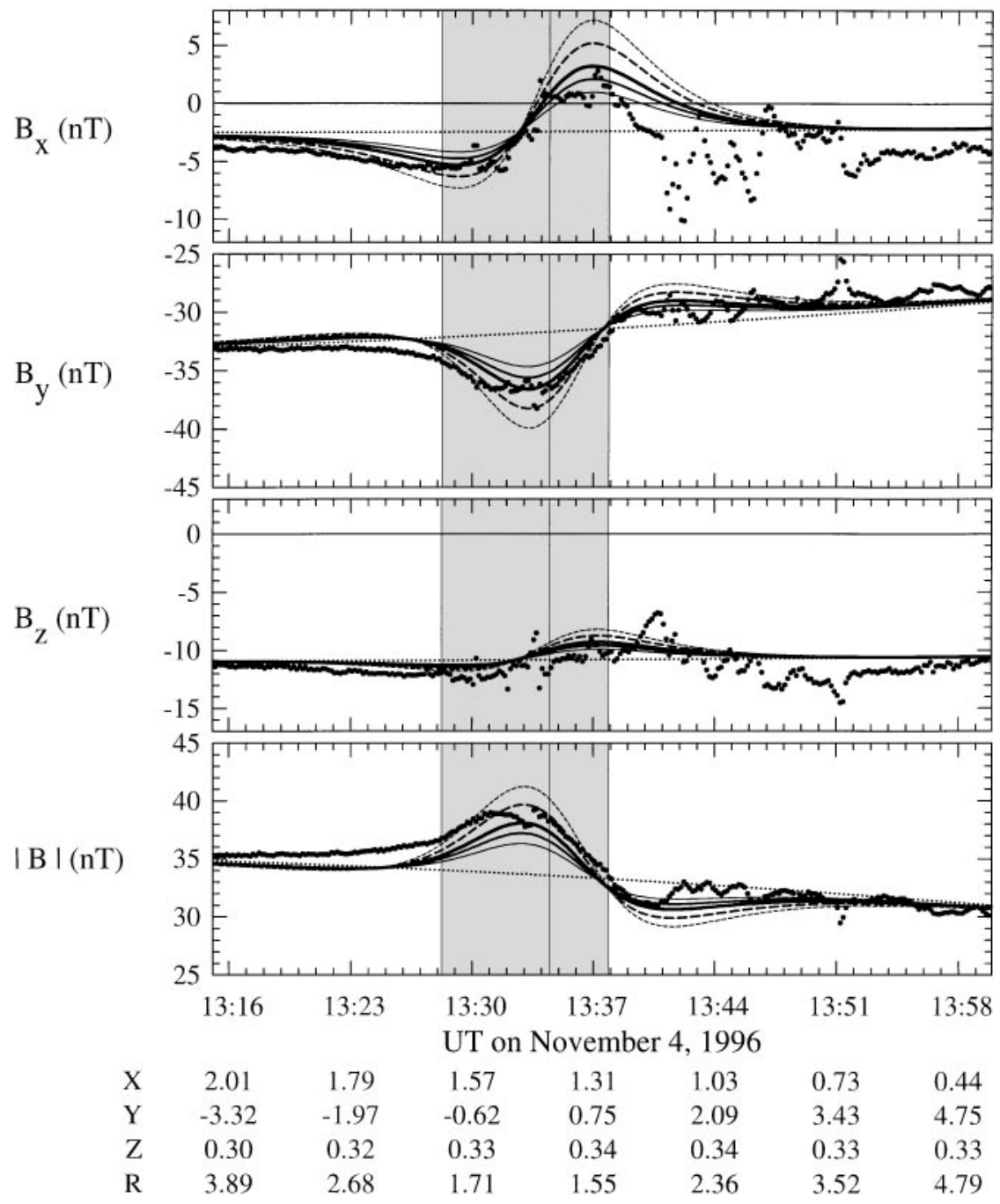

FIG. 3. Observed and modeled fields for the Callisto (a) flyby C3 on November 4, 1996 and (b) flyby C9 on June 25, 1997, in the coordinate system defined in Section 4.1.1. Thick dots show the measured field averaged over 10-s intervals. Solid and dashed curves show the predicted field for different amplitudes $A$ of the induced field and no phase lag $(\phi=0): A=0.6$ (thinnest solid curve), $A=0.8$ (solid curve of intermediate thickness), $A=1$ (thickest solid curve), $A=1.35$ (thin dashed curve), and $A=1.7$ (thick dashed curve). The dotted trace shows the background field (equivalent to a model field with $A=0$ ). The gap between the data and the background field at the edges of the time window displayed here would disappear if the window size were increased. Below the time labels are given the $x, y, z$ coordinates of the spacecraft and the normalized distance $R=\sqrt{x^{2}+y^{2}+z^{2}}$ from Callisto's center. The solid vertical line indicates the time of closest approach. The shaded region indicates when the spacecraft was in the geometrical wake of Callisto (i.e., where $\sqrt{y^{2}+z^{2}} \leq 1$ and $x \geq 0$ ).

This is possible if the encounter occurs at a time $t_{0}$ when the primary field $\mathbf{B}_{\text {prim }}$ is weakening $\left(d\left|\mathbf{B}_{\text {prim }}\right| / d t<0\right)$, or more generally if $\mathbf{B}_{\text {prim }}$ was stronger at some time $t_{1}$ during the $1 / 4$ wave period preceding closest approach, i.e., the time interval $\left[t_{0}-\right.$ $\left.\pi /(2 \omega), t_{0}\right]$. In this case, there exists a phase lag $\phi=\omega\left(t_{1}-t_{0}\right)$ between $0^{\circ}$ and $90^{\circ}$, for which both $\mathbf{B}_{\text {prim }}\left(t=t_{0}-\phi / \omega=t_{1}\right)$ and $\mathbf{B}_{\mathrm{sec}, \infty}\left(t=t_{0}-\phi / \omega=t_{1}\right)$ are stronger than $\mathbf{B}_{\text {prim }}\left(t=t_{0}\right)$ and $\mathbf{B}_{\mathrm{sec}, \infty}\left(t=t_{0}\right)$, respectively. It follows from Eq. (7) that the induced field $\mathbf{B}_{\mathrm{sec}}\left(t=t_{0}\right)$ is then larger than it would have been for $\phi=0$ and the same amplitude $A$.

To assess the importance of this effect and attempt to determine the phase lag from the observations, we need to evaluate
$\mathbf{B}_{\text {prim }}(t)$ during the time interval $\pi /(2 \omega)=2.55 \mathrm{~h}$ before each encounter. For this purpose, we used the Khurana (1997) model of the background field, and adjusted its parameters to obtain a good fit to magnetic field measurements taken over a few synodic periods before each individual encounter. During the $2.55 \mathrm{~h}$ preceding closest approach on encounters $\mathrm{C} 3$ and $\mathrm{C} 9$, the magnitude of the model primary field did not exceed its closest approach value by more than $10 \%$ and $20 \%$, while its direction did not differ by more than $6^{\circ}$ and $9^{\circ}$, respectively (see Table I). This stability of the primary field is a consequence of the thin current sheet, as already discussed in Section 2, and the timing of encounters $\mathrm{C} 3$ and $\mathrm{C} 9$, which both took place 
b $\quad$ C9 data \& models for $A=0,0.6,0.8,1,1.35,1.7$ and $\phi=0$

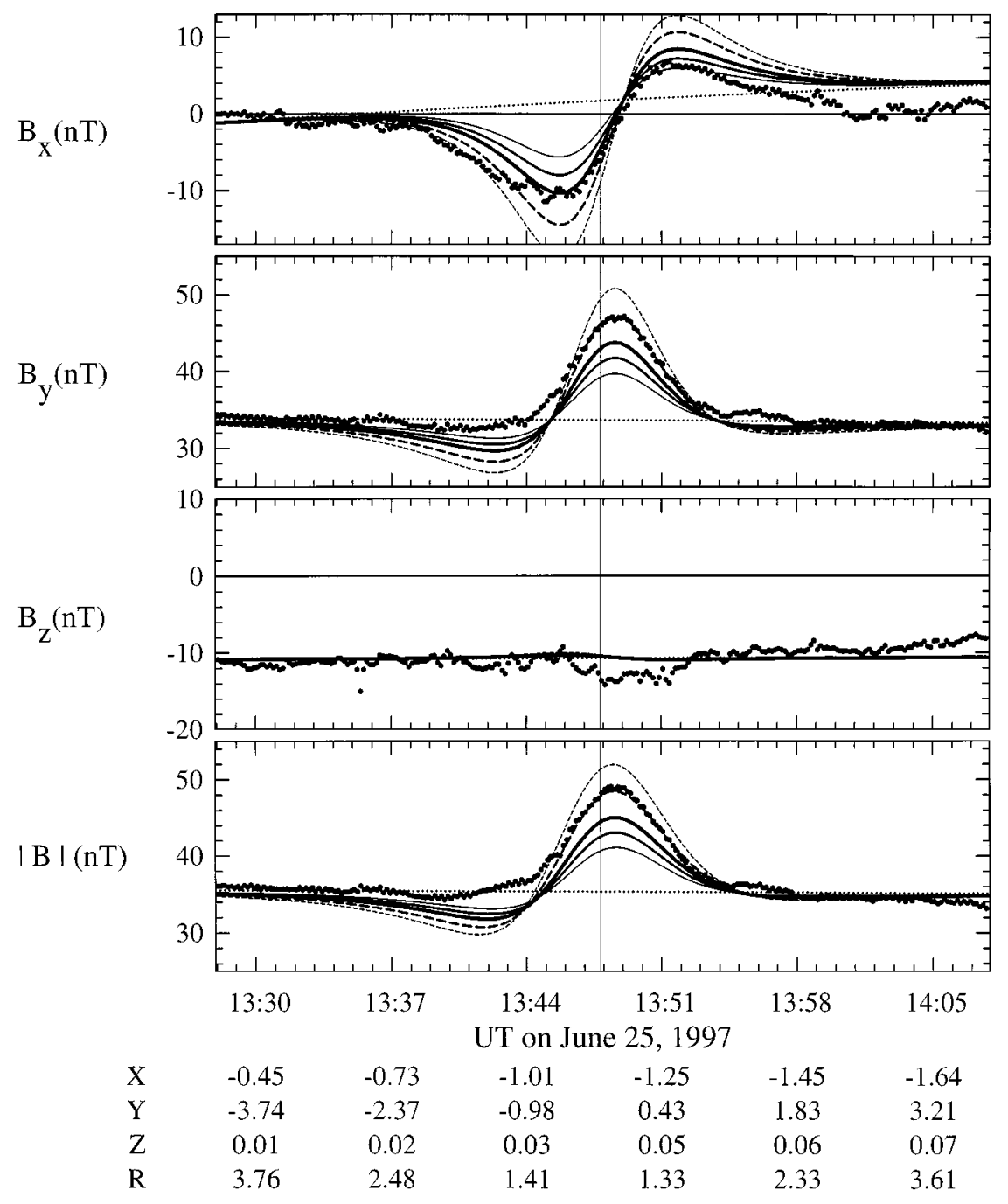

FIG.3- Continued

toward the end of the relatively long time intervals when the moon was well outside of the current sheet (see Fig. 1b of Kivelson et al. 1999). Because the primary field hardly varied during the interval $\left[t_{0}-2.55 \mathrm{~h}, t_{0}\right]$, the effect of any phase lag on the predicted induction signature is small. We verified that a $6^{\circ}$ change in the direction of the induced dipole moment would not significantly alter the match between the induced field model and the observed field, considering the uncertainties in the determination of the background field and distortions due to local plasma currents. It thus appears unfortunately not possible to determine the phase lag from these data without better modeling of the magnetospheric environment and the moonplasma interaction. Regarding the constraints on the amplitude $A$, however, it is sufficient to use the fact that for $\mathrm{C} 3$ the "retarded" primary field $\mathbf{B}_{\text {prim }}\left(t=t_{0}-\phi / \omega\right)$ from the model is almost in the same direction as the nonretarded $\mathbf{B}_{\text {prim }}\left(t_{0}\right)$, and that its magnitude does not exceed $B_{\text {prim }}\left(t_{0}\right)$ by more than $10 \%$ for any phase lag $\phi\left(0<\phi \leq 90^{\circ}\right)$, as mentioned above. We therefore conclude that the uncertainly in the phase lag does not extend the range of possible amplitudes $A$ estimated in Section 4.1.1 by more than $\pm 10 \%$. Since $A$ is smaller than 1 if the medium outside the moon is not conducting (to be justified in Section 6), the range of acceptable values of $A$, irrespective of assumptions about the phase lag, falls within the range 0.7 to 1 .

\subsection{Observations at Europa}

Up to October 1999, Galileo made close flybys of Europa on its 4th, 6th, 11th, 12th, and 14th through 19th orbits around Jupiter (labeled E4, E6, E11, E12, E14-E19). No magnetometer data were acquired during passes E6, E16, and E18. The data from Europa encounters up to E14 have already been presented by Kivelson et al. (1998). During encounters E11, E12, E15, $\mathrm{E} 17$, and E19, the measured field agrees poorly with induced 
TABLE I

The Background and the Primary Fields at E uropa and C allisto before and during Encounters C 3, C9, E 4, and E 14

From Khurana (1997) model

\begin{tabular}{|c|c|c|c|c|c|c|c|}
\hline & & \multirow[b]{3}{*}{$\begin{array}{c}\text { Field } \\
\text { component }^{a}\end{array}$} & \multirow{2}{*}{\multicolumn{2}{|c|}{ From Galileo data }} & \multirow[b]{3}{*}{$\begin{array}{c}\mathbf{B}_{\text {prim, C/A }} \\
(\mathrm{nT})^{d}\end{array}$} & \multirow{2}{*}{\multicolumn{2}{|c|}{ For times $t$ during the $1 / 4$ synodic period before $\mathrm{C} / \mathrm{A}$}} \\
\hline \multirow[b]{2}{*}{ Encounter } & & & & & & & \\
\hline & & & $\begin{array}{c}\mathbf{B}_{\mathrm{J}} \text { at C/A } \\
(\mathrm{nT})^{b}\end{array}$ & $\frac{\mathbf{B}_{\text {prim, }, \mathrm{C} / \mathrm{A}}}{(\mathrm{nT})^{c}}$ & & $\begin{array}{c}\text { Max. angle } \\
\left(\mathbf{B}_{\text {prim }}(t), \mathbf{B}_{\text {prim,C/A }}\right)^{e}\end{array}$ & $\begin{array}{c}\text { Max. }(\min .) \text { of } \\
B_{\text {prim,C/A }}-B_{\text {prim }}(t)(\mathrm{nT})^{f}\end{array}$ \\
\hline \multirow[t]{6}{*}{ Callisto } & $\mathrm{C} 3$ & $B_{x}$ & -2.4 & -2.4 & -3.4 & & \\
\hline & & $B_{y}$ & -31.7 & -31.7 & -33.4 & $5.5^{\circ}$ & $3.2(-3.0)$ \\
\hline & & $B_{z}$ & -10.8 & 0 & -0.3 & & \\
\hline & $\mathrm{C} 9$ & $B_{x}$ & 1.7 & 1.7 & 5.8 & & \\
\hline & & $B_{y}$ & 33.65 & 33.65 & 33.9 & $8.6^{\circ}$ & $2.2(-7.5)$ \\
\hline & & $B_{z}$ & -10.7 & 0 & -1.9 & & \\
\hline \multirow[t]{6}{*}{ Europa } & $\mathrm{E} 4$ & $B_{x}$ & 53.4 & 53.4 & 49.8 & & \\
\hline & & $B_{y}$ & -176.2 & -176.2 & -172.0 & $148.5^{\circ}$ & $0(-104.0)$ \\
\hline & & $B_{z}$ & -410.0 & 0 & -21.0 & & \\
\hline & E14 & $B_{x}$ & 9.6 & 9.6 & 10.1 & & \\
\hline & & $B_{y}$ & -212.2 & -212.2 & -213.2 & $132.5^{\circ}$ & $0(-138.1)$ \\
\hline & & $B_{z}$ & -402.7 & 0 & -17.6 & & \\
\hline
\end{tabular}

Note. This table lists values used in assessing the background field $\mathbf{B}_{\mathrm{J}}$, the primary field at closest approach $\mathbf{B}_{\text {prim, }} / \mathrm{A}$, and the temporal variation of the primary field $\mathbf{B}_{\text {prim }}(t)$ before each of the considered encounters. For the construction of the model fields of Figs. 3-6, $\mathbf{B}_{\mathrm{j}}$ and $\mathbf{B}_{\text {prim,C/A }}$ were estimated using Galileo data (see Sections 4.1.1 and 4.2). For the analysis of the phase lag (Sections 4.1.2 and 4.2.2), $\mathbf{B}_{\text {prim,C/A }}$ and $\mathbf{B}_{\text {prim }}(t)$ were determined from the Khurana (1997) model $\left(\mathbf{B}_{\text {prim }}(t)=\left\langle\mathbf{B}_{\mathrm{J}}\right\rangle-\mathbf{B}_{\mathbf{J}}(t)\right.$, where $\mathbf{B}_{\mathrm{J}}(t)$ is the model jovian background field predicted at the moon's position at time $t$ and the brackets denote an average over many synodic periods).

${ }^{a}$ All field components are given in the moon-centered coordinate systems defined in Section 4.1.1 for Callisto and 4.2 for Europa.

${ }^{b}$ Jovian background field at the location of the moon at the time of closest approach,. estimated from Galileo measurements (see Section 4.1.1).

${ }^{c}$ Primary field at closest approach, estimated from the equatorial projection of the background field at closest approach given by the previous column.

${ }^{d}$ Primary field at closest approach, estimated from the Khurana (1997) model. Note that it does not differ appreciably from the primary field estimated using Galileo data given in the previous column. In particular, the component $B_{z}$ is small compared to $B_{x}$ and $B_{y}$, as expected (see Section 2).

${ }^{e}$ Largest angular separation between the primary field at closest approach and the primary field during the preceding $1 / 4$ synodic period.

${ }^{f}$ Largest (smallest) algebraic difference between the magnitude of the primary field at closest approach and the magnitude of the primary field during the preceding $1 / 4$ synodic period.

dipole models and exhibits short-scale fluctuations, probably due to plasma currents, with amplitudes comparable or larger than the perturbations expected from an induced field. Encounters E12 and E15 occurred while Europa was very close to the center of the dense plasma sheet (see Fig. 1a of Kivelson et al. 1998). On flybys E11, E17, and E19, the spacecraft was almost $1 R_{\mathrm{E}}$ or more away from the equatorial plane, where disturbances due to the plasma currents from the Alfvén wings are expected to be strong (e.g., Neubauer 1998). We will therefore restrict our attention here to the remaining encounters E4 and E14, which are closer to the equatorial plane (within less than 0.1 and $0.5 R_{\mathrm{E}}$, respectively) and took place in the lobes, well outside of the jovian current sheet (see Figs. 3 and 1a of Kivelson et al. 1999).

Figures $4 \mathrm{a}$ and $4 \mathrm{~b}$ show the magnetic field $\mathbf{B}_{\text {obs }}$ measured during the two Europa encounters E4 and E14, respectively, as well as induced field models $\mathbf{B}_{\text {tot }}$ (for various values of $A$ and for $\phi=0$ ) and the background field $\mathbf{B}_{\mathrm{J}}$. All fields were constructed in the same manner as for Callisto (see Section 4.1.1). The coordinate system is analogous to that defined for Callisto in Section 4.1.1, but has its origin at Europa's center and distances are measured in Europa radii $R_{\mathrm{E}}$. It is apparent that the equatorial components of the field ( $B_{\mathrm{x}}$ and $B_{\mathrm{y}}$ ) are in rough agreement with induced field models having $\phi=0$ and $A$ close to 1 . However, large departures from the models are evident in the vertical $B_{\mathrm{z}}$ component and the magnitude $B$. During flyby E14, the measured field was consistently stronger than both the background and the induced field models and reached its maximum strength near closest approach. A gradual increase in field strength was measured starting as far as approximately $8 R_{\mathrm{E}}$ away from the moon on the inbound leg. A more abrupt decrease was observed on the outbound leg, with $B$ falling back to background values at a distance of less than $4 R_{\mathrm{E}}$. The field increase at large distances is indicative of currents flowing in the ambient plasma rather than a dipolar (or higher order multipole) field generated by Europa. The MHD simulation of the Europa interaction by Kabin et al. (1999) and their comparison with E4 data confirm that the $B_{\mathrm{z}}$ perturbation can wholly be attributed to plasma currents, as was suggested by Kivelson et al. (1999). The compression of the field on the upstream side $(x<0)$ is qualitatively consistent with deceleration of the corotating flow by the obstacle and increasing mass-loading closer to Europa. On the downstream side $(x>0)$ the field strength has almost returned to its background value. On the E4 flyby, the field strength signature is dominated by rapid (less than $5 \mathrm{~min}$ ) or small-scale 
(less than $1 R_{\mathrm{E}}$ ) fluctuations too abrupt to be caused by internal fields and probably due at least in part to the temporal variability of the plasma environment. In contrast to the situation at $\mathrm{Cal}-$ listo, the discrepancy between the observed and predicted field magnitude $B$ is of the same order or larger than the predicted perturbations from induction in the $B_{x}$ and $B_{y}$ components. We therefore attempt to correct for the effect of plasma currents in the next section.

4.2.1. Approximate correction for plasma effects. As a firstorder method to take into account plasma disturbances, we employ a technique suggested by Khurana et al. (1998). If the small inclination of $\mathbf{B}_{\mathrm{J}}$ along $x\left(<8^{\circ}\right.$ for both $\mathrm{E} 4$ and E14, as can be verified from Table I) is ignored, and if we assume that the ambient plasma is equally distributed along the field northward and southward of Europa, then the system can be considered as roughly symmetric about a plane passing through the moon's center and perpendicular to $\mathbf{B}_{\mathrm{J}}$ (ignoring the effect of the induced dipole moment, which tends to break the symmetry as shown by Neubauer 1999). In this symmetry plane, the magnetic perturbations due to plasma currents should be purely compressional and not involve any bending of the field. Galileo remained within less than $1 R_{\mathrm{E}}\left(0.5 R_{\mathrm{E}}\right)$ of this plane between 06:41 and 07:19 UT (06:51 and 07:10) on encounter E4, and between 13:06 and 13:30 UT (13:12 and 13:24) on encounter E14. Thus, during these time intervals, where the perturbations predicted from induction models are large, the local plasma currents should not contribute significantly to changes in the field direction. To the extent that the field $\delta \mathbf{B}$ generated by these currents can be treated as a small perturbation to the field $\mathbf{B}_{\text {tot }}$, the sum of Jupiter's background field $\mathbf{B}_{\mathbf{J}}$, and Europa's inductive response $\mathbf{B}_{\mathrm{sec}}$, this implies

$$
\delta B_{x} / B_{x, \text { tot }}=\delta B_{y} / B_{y, \text { tot }}=\delta B_{z} / B_{z, \text { tot }}=\delta|\mathbf{B}| / B_{\text {tot }},
$$

where $\delta|\mathbf{B}|=\left|\mathbf{B}_{\text {obs }}\right|-\left|\mathbf{B}_{\text {tot }}\right|$ is the difference of magnitudes between the model field $\mathbf{B}_{\text {tot }}$ and the observed field $\mathbf{B}_{\text {obs. }}$. Thus from Eq. (8) and the measured field magnitude, we can infer the three components of the plasma perturbation $\delta \mathbf{B}$ near the abovementioned symmetry plane. We obtain model fields corrected for plasma effects by adding $\delta \mathbf{B}$ to $\mathbf{B}_{\text {tot }}$.

In Figs. 5a and 5b, the model fields, now corrected for plasma effects, are once again plotted with the observed field. It is apparent from a comparison with Figs. $4 \mathrm{a}$ and $4 \mathrm{~b}$ that the corrected models improve the fit to the $B_{y}$ component of the signature, while the fit to the $B_{x}$ component does not deteriorate. Since our plasma correction consisted of imposing the observed field magnitude, the agreement between the predicted and observed $B_{z}$ (which dominates in magnitude over $B_{x}$ and $B_{y}$ ) is an automatic byproduct of the correction and has no significance. The remaining discrepancy between the observed and modeled $B_{x}$ and $B_{y}$ may be due to the finite distance between the spacecraft and the symmetry plane, or to the breaking of symmetry caused by the induced fields, as mentioned above, an unsymmetrical distribution of plasma northward and southward of the moon, or the small $x$ component of the background field. Nevertheless, we conclude from Figs. 5a and 5b that the observed field signatures are roughly consistent with an inductive response characterized by a phase lag $\phi=0$ and an amplitude $A$ in the range of 0.7-1.6. This range reduces to $0.7-1$ if the conductivity outside the moon is negligible, as we will argue in Section 6.

4.2.2. Phase lag. Contrary to the case of Callisto described in 4.1.2, the primary field experienced by Europa varies strongly during the whole time interval between consecutive current sheet crossings (see Section 2 and Fig. 1a of Kivelson et al. 1999). Using the Khurana (1997) field model with parameters adapted to fit magnetic field observations several synodic periods before each flyby, we estimate that the primary field direction varied by roughly $150^{\circ}$ and $130^{\circ}$ over the $2.8 \mathrm{~h}(=1 / 4$ synodic period at Europa) before encounters E4 and E14, respectively (see Table I). With an inductive phase lag $\phi>0$, the dipole moment could thus point in a direction substantially different from that of the instantaneous primary field, a rotation that would be clearly visible in the data. In order to constrain the phase lag, we therefore allow the induced dipole moment $\mathbf{M}$ to rotate in the equatorial plane (while keeping $M_{z}=0$ ) to an angle $\alpha$ measured from the direction of the dipole moment for $\phi=0$ (i.e., the direction of $-\mathbf{B}_{\text {prim, C/A }}$; see Section 3), maintaining the amplitude $A=1$. Over time, the primary field rotates clockwise in the $\left(B_{x}, B_{y}\right)$ plane and we take $\alpha$ positive for an anti-clockwise deviation of M. Thus, a positive phase lag $\phi$ implies that $\mathbf{M}$ is rotated by a positive angle $\alpha$. Note that the angles $\alpha$ and $\phi$ are different because the primary field is not circularly polarized, since the oscillation amplitude of $B_{y}$ is larger than that of $B_{x}$ (see Section 2 and Fig. 1 of Khurana et al. 1998). In Figs. 6a and 6b we compare the observations, corrected for plasma compression as described in the previous section, to the induced field models for various angles $\alpha$ and $A=1$. Since nonzero angles $\alpha$ correspond to nonzero phase lags and are thus physically inconsistent with our assumed amplitude $A=1$, only the phasing of the model fields (i.e., the timing of the increases and decreases in components $B_{x}$ and $B_{y}$ ) for $\alpha \neq 0$ should be compared to the observed signature, and not the amplitudes of the model field. It can be seen that the $B_{y}$ component is reasonably well reproduced with $(A, \alpha)=\left(1,0^{\circ}\right)$. The $B_{x}$ component on the other hand is more consistent with $\alpha<0^{\circ}$ on E4 and more consistent with $\alpha>0^{\circ}$ on E14. A negative angle $\alpha$ is unphysical since it corresponds to a negative phase lag $\phi$ (i.e., a secondary field leading the primary field). In view of the contradictory indications of $B_{x}$, the transverse component expected to be most affected by the moon-plasma interaction as the field lines are draped around the obstacle in the flow direction, we refrain from interpreting the residual phase discrepancy in $B_{x}$ as an electromagnetic phase delay.

Concerning the estimates of $A$, however, it is sufficient to note that the Khurana (1997) field model predicts that the equatorial component of Jupiter's field was monotonically increasing in magnitude during the 2.8 -h interval before both encounters E4 and E14 (see Table I). As a consequence, if a phase lag $\phi>0$ 


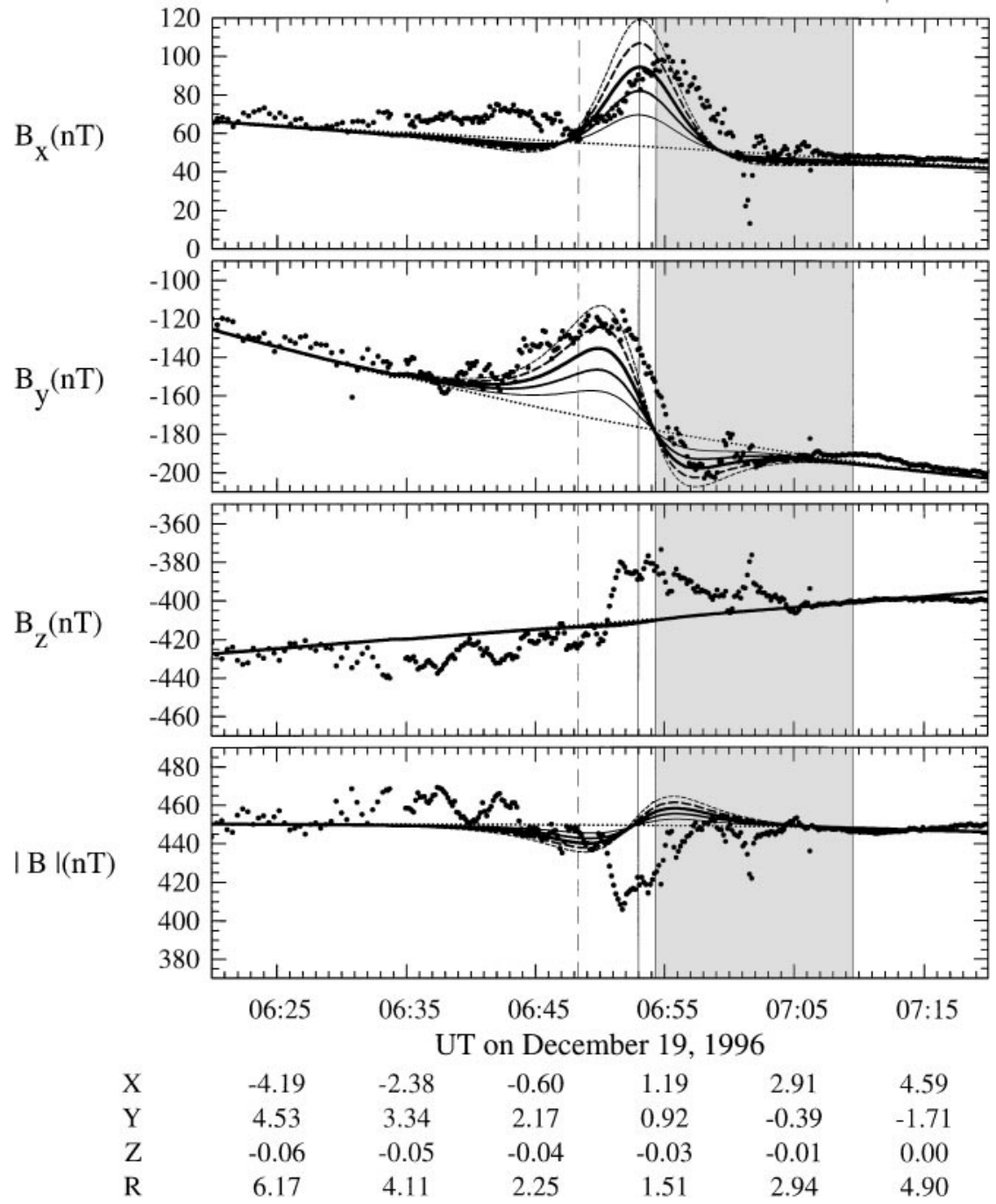

FIG . 4. Observed and modeled fields for the Europa (a) flyby E4 on December 19, 1996 and (b) flyby E14 on March 29, 1998, in the coordinate system defined in Section 4.2. Thick dots show the measured field averaged over 10 or $24 \mathrm{~s}$. Solid and dashed curves show the predicted field for different amplitudes $A$ of the induced field and no phase lag: $A=0.4$ (thinnest solid curve), $A=0.7$ (solid curve of intermediate thickness), $A=1$ (thickest solid curve ), $A=1.3$ (dashed thin), and $A=1.6$ (dashed thick). The dotted curve shows the background field (equivalent to a model field with $A=0$ ). The gap between the data and the background field at the edges of the time window shown would disappear if the window size were increased. Below the time labels are given the $x, y, z$ coordinates of the spacecraft and the normalized distance $R=\sqrt{x^{2}+y^{2}+z^{2}}$ from Europa's center. The solid vertical line indicates the time of closest approach. The shaded region indicates when the spacecraft was in the geometrical wake of Europa $\sqrt{y^{2}+z^{2}} \leq 1$ and $x \geq 0$ ). The dashed vertical line marks the passage through the prime meridian of Europa, $x=0$.

were present, the induced field $\mathbf{B}_{\mathrm{sec}}\left(t_{0}\right)$ would be weaker than for $\phi=0$, and thus weaker than required by the observations, unless the amplitude $A$ were even higher than proposed in 4.2.1 (see Eq. 7). The range of acceptable values for $A$ is therefore included in 0.7-1, and even narrower. We note that the strength of the dipole moment favored by Kabin et al. (1999) corresponds to a normalized amplitude of the induced field of 0.7 .

On the basis of the two encounters E4 and E14 alone, it is not possible to rule out the alternative possibility of a permanent dipole moment oriented in roughly the same direction and with the same strength as the induced dipole moment predicted. This ambiguity remains because the orientation and strength of the jovian background field were very similar during the E4 and E14 flybys (see Fig. 1a of Kivelson et al. 1999), leading to little difference in the induced dipole moments predicted. However, a strong test of the induction model was provided by the recent flyby E26 on January 3, 2000, during which the jovian field had an orientation roughly opposite to that during encounters E4 and E14. Kivelson et al. (2000) showed that the observed field perturbations are indeed in much better agreement with the induced dipole model than with a permanent dipole moment capable of accounting for the E4 and E14 data. 
b E14: data \& models for $\mathrm{A}=0,0.4,0.7,1,1.3,1.6$ and $\phi=0$
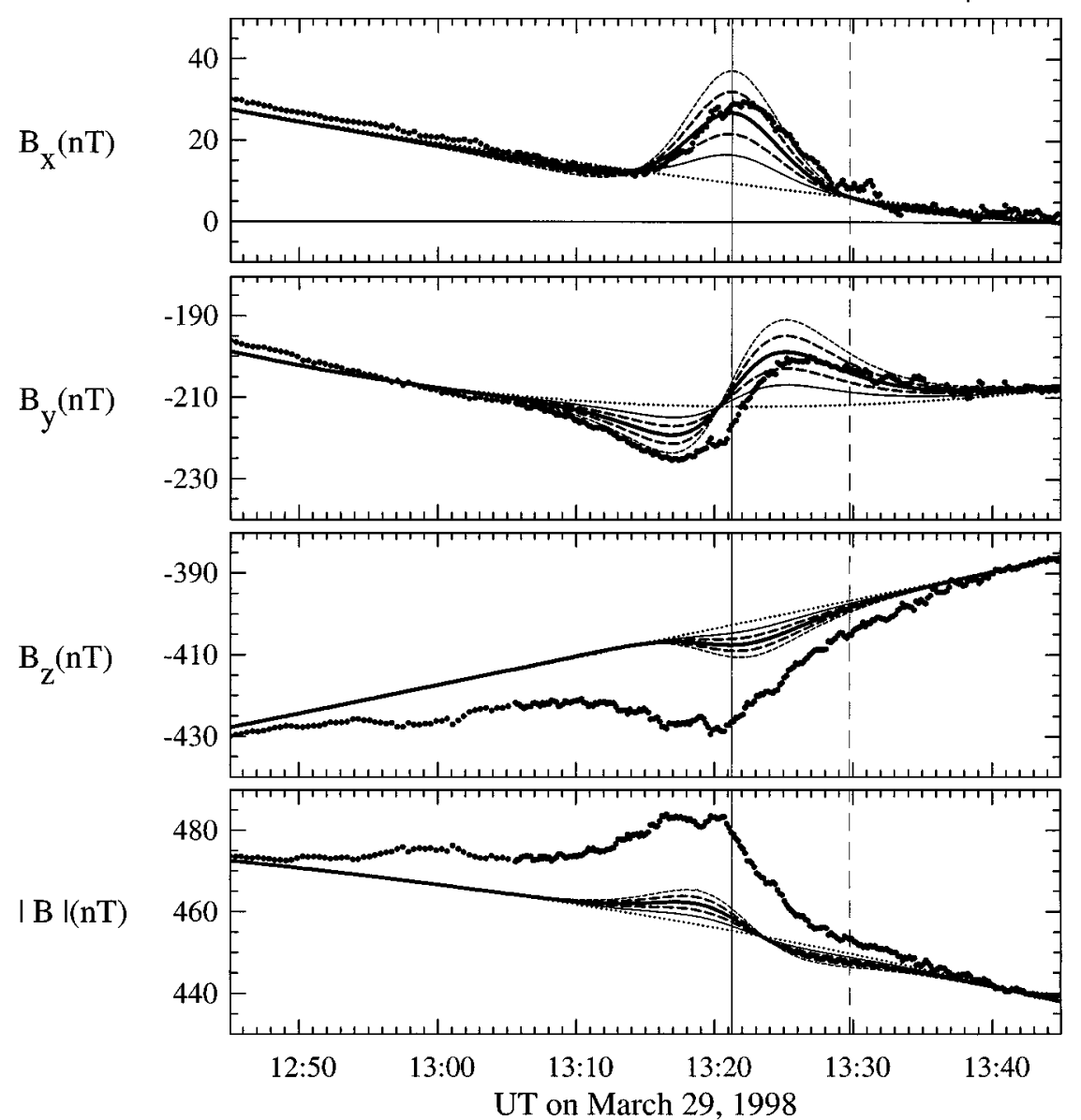

$\begin{array}{lcccccc}\mathrm{X} & -6.60 & -4.98 & -3.34 & -1.67 & 0.03 & 1.75 \\ \mathrm{Y} & 4.36 & 2.53 & 0.69 & -1.15 & -2.96 & -4.74 \\ \mathrm{Z} & 0.34 & 0.37 & 0.40 & 0.42 & 0.43 & 0.44 \\ \mathrm{R} & 7.92 & 5.59 & 3.43 & 2.08 & 2.99 & 5.07\end{array}$

FIG. 4-Continued

\section{CONSEQUENCES ON THE ELECTRICAL AND PHYSICAL STRUCTURE OF EUROPA AND CALLISTO}

The interior structures of Europa and Callisto are still poorly constrained. Both moons are known to be covered by solid water ice and their average densities require both $\mathrm{H}_{2} \mathrm{O}$ and silicates to be present in their interiors. Using gravitational measurements from Galileo flybys E4 and E6, Anderson et al. (1998a) have inferred that Europa possesses an $\mathrm{H}_{2} \mathrm{O}$ crust $100-200 \mathrm{~km}$ thick overlying a silicate mantle, which may in turn surround a metallic core of radius $0.4 R_{\mathrm{E}}$. A similar analysis of Galileo gravity data on passes C3, C9, and C10 by Anderson et al. (1998b) indicated that Callisto, long considered undifferentiated, consists in fact of a partially differentiated mixture of rock and ice, possibly (but not necessarily) with an $\mathrm{H}_{2} \mathrm{O}$ crust less than $350 \mathrm{~km}$ thick. A metallic core of radius larger than $0.25 R_{\mathrm{C}}$ was ruled out by the authors. It has been speculated (e.g., Squyres et al. 1983) that the ice layer of Europa may be partially melted by the heat generated through tidal flexing and radioactive decay. The state of the $\mathrm{H}_{2} \mathrm{O}$ layer cannot be determined from gravitational measurements because the densities of liquid water and ice are too close. The recent observation of topographic features by Galileo, however, has provided evidence for a liquid or viscous medium underlying a thin ice crust in the more or less recent geological past (Carr et al. 1998, Pappalardo et al. 1998, Rathbun et al. 1998, Hoppa et al. 1999). As we will discuss below, a sensitive indication of the state of the water at the present epoch is given by its electrical conductivity.

We now combine the observational characteristics of the induced fields determined in Section 4 with the theoretical results of Section 3 in order to constrain the first-order conductivity structure of the two moons. We thus assume that the electrical structure of both Europa and Callisto can be represented by a uniform conducting shell with insulating interior and surroundings. Other conducting regions not allowed for by this model will be discussed in Section 6. 


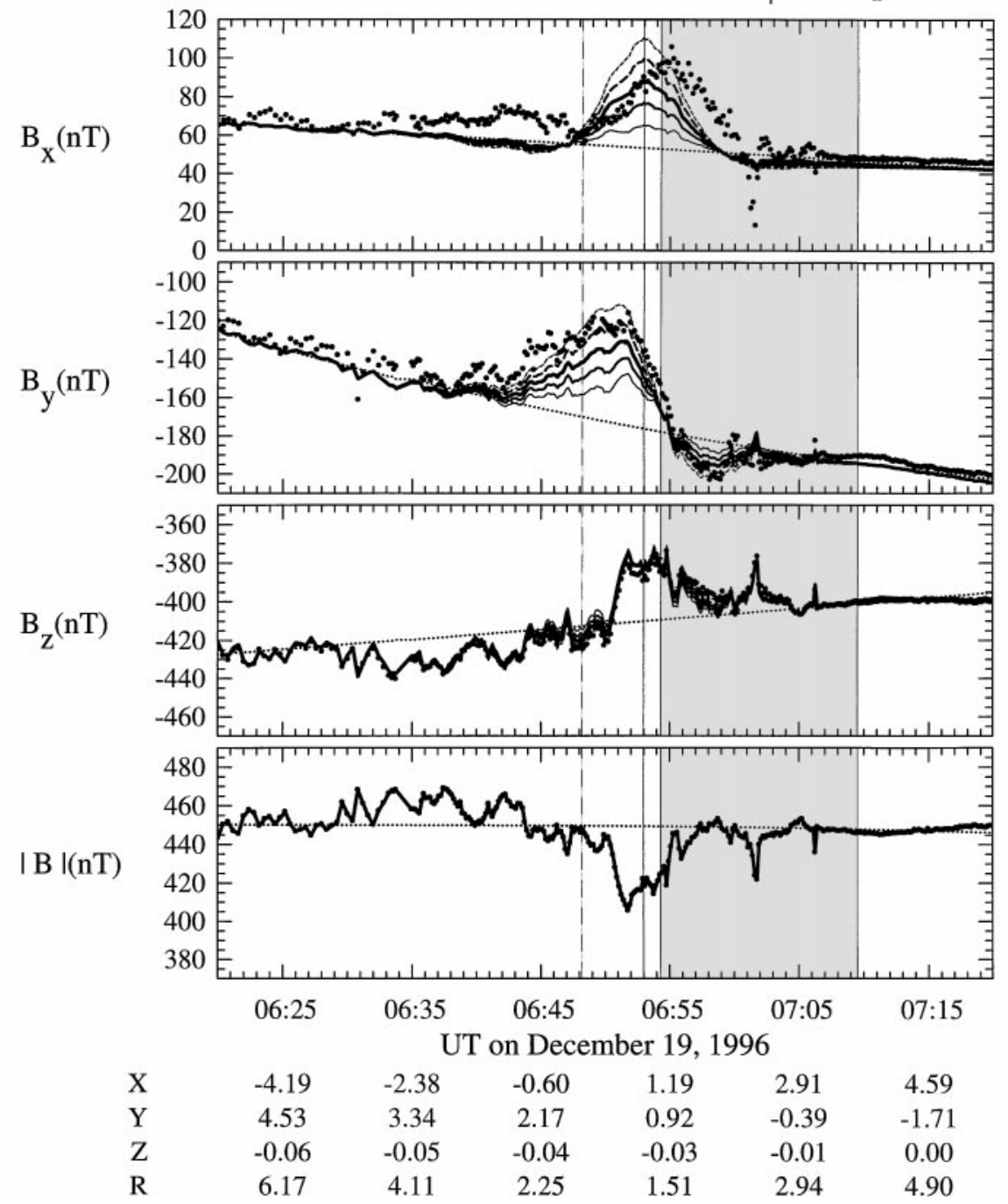

FIG. 5. (a) Same as that described in the legend to Fig. 4a, but with the model fields corrected for plasma disturbances (see Section 4.2.1). The agreement between the predicted and observed field magnitude $|B|$ and the $B_{z}$ component has no significance (see text). (b) Same as (a), but for Europa flyby E14 on March 29, 1998.

The reference conductivity $\sigma_{\mathrm{m}}$ defined in Section 3 is equal to $4.2 \mathrm{mS} / \mathrm{m}$ for Europa and $1.6 \mathrm{mS} / \mathrm{m}$ for Callisto. The strong inductive response apparent in the Galileo data (Section 4) implies that the moons must possess regions of conductivities much larger than $\sigma_{\mathrm{m}}$ (Section 3 ). For a given amplitude $A$, a lower limit on the conductivity can be read from Fig. 2 by considering an arbitrarily thick conducting shell. For $A>0.7$, Europa's shell must have a conductivity $\sigma>58 \mathrm{mS} / \mathrm{m}$. For Callisto, $A>0.7$ requires a shell conductivity $\sigma>22 \mathrm{mS} / \mathrm{m}$. We tabulated the minimum conductivities for selected values of $A$ in Table II.

The electrical conductivity of a material is highly dependent on its state. The conductivity of pure solid ice is many orders of magnitude smaller than the values required above. Contamination and the presence of acids can increase the conductivity of ice by orders of magnitude. However conductivities of
$10 \mathrm{mS} / \mathrm{m}$ or larger can be achieved only if the temperature is close enough to the melting point that the ice is partially melted (Keller and Frischknecht 1996). For a melt fraction $q$ below 0.2, the bulk conductivity $\sigma$ follows Archie's law $\sigma / \sigma_{\text {liq }}=C q^{n}$, where $\sigma_{\text {liq }}$ is the conductivity of the melt, $C$ is a coefficient smaller than 0.3 , and the exponent $n$ is larger than 1.3 (Watanabe and Kurita 1993). If the melt has the conductivity of Earth seawater $\left(\sigma_{\text {liq }}=2.75 \mathrm{~S} / \mathrm{m}\right.$, after Montgomery 1963$)$, a bulk conductivity of 60 and $20 \mathrm{mS} / \mathrm{m}$ thus implies a rather substantial melt fraction $q>13$ and 5\%, respectively.

The magnitude of the inductive response can easily be explained by a liquid ocean of salty water with a conductivity of $2.75 \mathrm{~S} / \mathrm{m}$. This conductivity is very close to the $2.5 \mathrm{~S} / \mathrm{m}$ estimated by Kargel and Consolmagno (1996) for a Europa ocean consisting of a eutectic solution of $\mathrm{MgSO}_{4}$. From Fig. 2, one 
b E14 data \& models for $\mathrm{A}=0,0.4,0.7,1,1.3,1.6$ and $\phi=0 \mathrm{w}$. plasma corr.

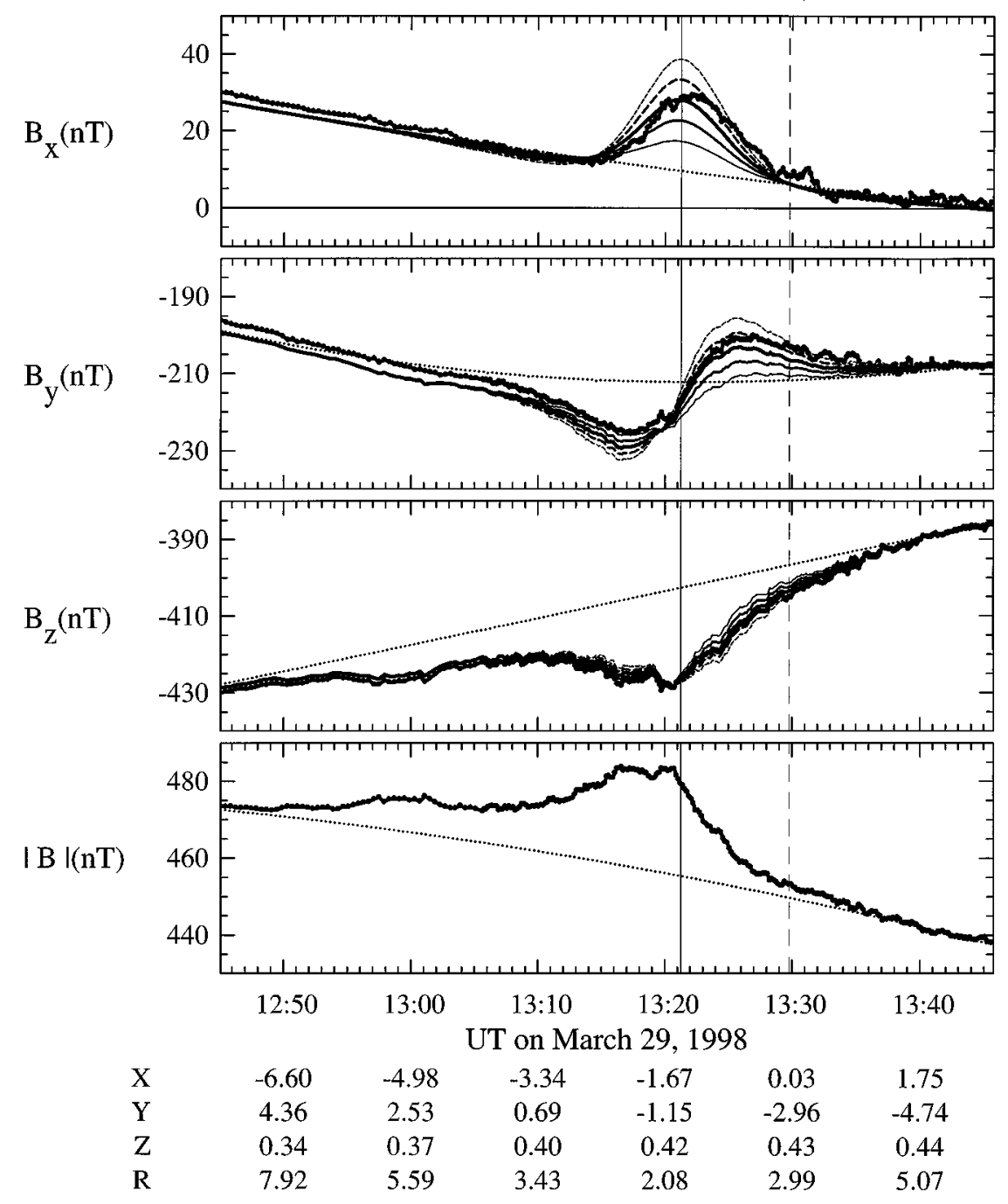

FIG. 5-Continued

can estimate the minimum required thickness of such an ocean. The minimum thicknesses for different values of $A$ are listed in Table II. For Europa, an Earth-like near surface ocean of $3.5 \mathrm{~km}$ thickness can account for an induced field of amplitude $A=0.7$. This agrees with the results that Kuramoto et al. (1998) obtained using a numerical solution of the induction problem. At Callisto, an induced field amplitude $A=0.7$ can be achieved with an ocean only $2 \mathrm{~km}$ thick. If the conductivity is higher, even thinner oceans are possible.

In deriving the minimum conductivities of 58 and $22 \mathrm{mS} / \mathrm{m}$ for Europa and Callisto respectively, we did not impose restrictions on the thickness of the conducting shell. However, the conducting layer cannot be larger than the $\mathrm{H}_{2} \mathrm{O}$ layer itself, unless the underlying medium is also conducting (a possibility that we ignore in this section, but will address in section 6). If we take this additional constraint into consideration, the required conductivities increase. For Europa, where the $\mathrm{H}_{2} \mathrm{O}$ layer cannot be more than $200 \mathrm{~km}$ thick (Anderson et al. 1998a), the required conductivity for $A>0.7$ increases to $72 \mathrm{mS} / \mathrm{m}$. If the $\mathrm{H}_{2} \mathrm{O}$ layer is only $100 \mathrm{~km}$ thick, the minimum conductivity roughly doubles to $116 \mathrm{mS} / \mathrm{m}$. For Callisto, where the $\mathrm{H}_{2} \mathrm{O}$ layer does not exceed $350 \mathrm{~km}$ thickness, the required conductivity for $A>0.7$ increases slightly to $26 \mathrm{mS} / \mathrm{m}$ (see Table II for other values of $A$ ).

Although we have assumed that the conducting layer reaches up to the surface of the moon, it is in reality separated from the surface by a solid ice crust of thickness $d$ that acts as an insulator. An upper limit on the thickness of the insulating ice crust arises immediately from the fact that $A$ cannot exceed $\left(r_{0} / r_{\mathrm{m}}\right)^{3}$, which implies that $d / r_{\mathrm{m}} \leq 1-A^{1 / 3}$. Table II indicates the maximum possible crust thickness $d$, or depth of the conducting outer layer, at Europa and Callisto for different values of $A$. With $A>0.7$ at Europa, the conducting layer cannot lie deeper than $175 \mathrm{~km}$ below the surface; for Callisto, $A>0.7$ implies a maximum depth 
a E4 data \& models for $\alpha=-50,-20,0,20,50 \mathrm{deg}$. and $\mathrm{A}=1 \mathrm{w}$. plasma corr.

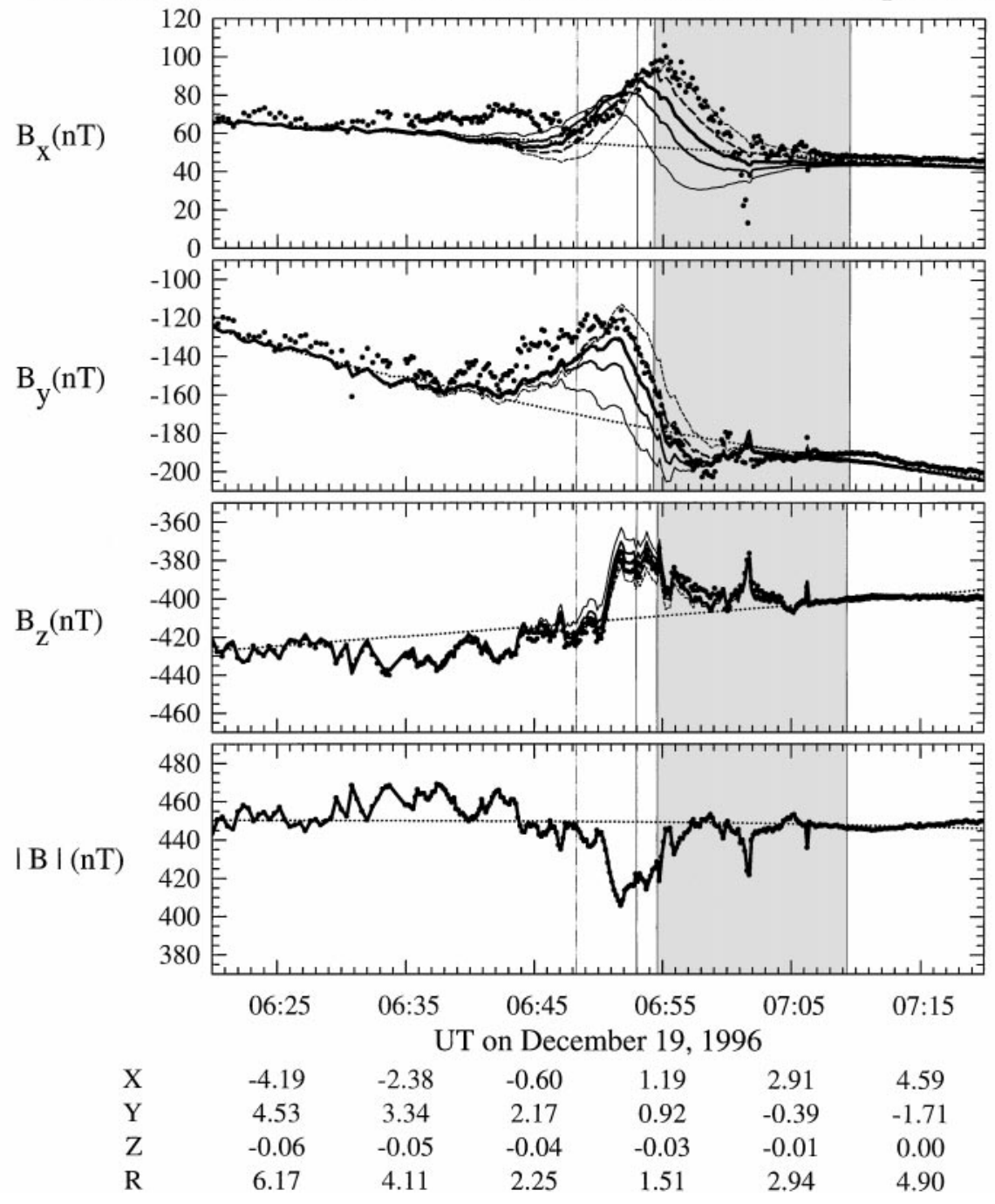

FIG. 6. (a) Similar to that described in the legend to Fig. 5a, but the modeled induced field dipoles have $A=1$ and various angles $\alpha$ with respect to the dipole orientation for $\phi=0^{\circ}: \alpha=50^{\circ}$ (thinnest solid line), $\alpha=20^{\circ}$ (solid line of internediate thickness), $\alpha=0^{\circ}$ (thickest solid line), $\alpha=-20^{\circ}$ (thin dashed line), and $\alpha=-50^{\circ}$ (thick dashed line). The dipole moments lay in the $x y$ plane and angles are counted positively in the anti-clockwise sense within this plane. Since the primary field rotates in the clockwise sense, a positive $\alpha$ corresponds to a positive inductive phase lag $\phi$. (b) Same as (a), but for Europa flyby E14 on March 29 , 1998.

of $270 \mathrm{~km}$. These limits must be satisfied even for a highly conducting shell $\sigma \gg \sigma_{\mathrm{m}}$. For smaller $\sigma$, the insulating crust must be even thinner. Unfortunately, our estimation of the inductive response $A$ is not precise enough to allow us to resolve ice layer thicknesses of the order of 1 or $10 \mathrm{~km}$, as suggested for Europa by Hoppa et al. (1999) and Rathbun et al. (1998), respectively.

\section{INFLUENCE OF A CONDUCTING MANTLE, CORE, IONOSPHERE, OR PICK-UP CLOUD}

In the previous section, we have assumed that the media above and below the $\mathrm{H}_{2} \mathrm{O}$ layer are insulating. We now discuss the role of a conducting silicate mantle or core below (Section 6.1) and the possibility of a conducting ionosphere or mass-loading region present above the surface of the moon (Section 6.2).

\subsection{Influence of a Conducting Core and/or Mantle}

Although a metallic core would effectively act as a perfect conductor (in Earth's core $\sigma \sim 3 \times 10^{5} \mathrm{~S} / \mathrm{m} \gg \sigma_{\mathrm{m}}$, e.g., Stacey 1992), it cannot by itself account for the observed inductive response, because its size is too small. To produce an induced field with $A=0.5$, for instance, the core would need to extend to $322 \mathrm{~km}$ below the surface of Europa, and $497 \mathrm{~km}$ for Callisto (see Table II). Such large cores are grossly inconsistent with the constraints from gravitational measurements mentioned in Section 5 . 
b E14 data \& models for $\alpha=-50,-20,0,20,50 \mathrm{deg}$. and $\mathrm{A}=1 \mathrm{w}$. plasma corr.

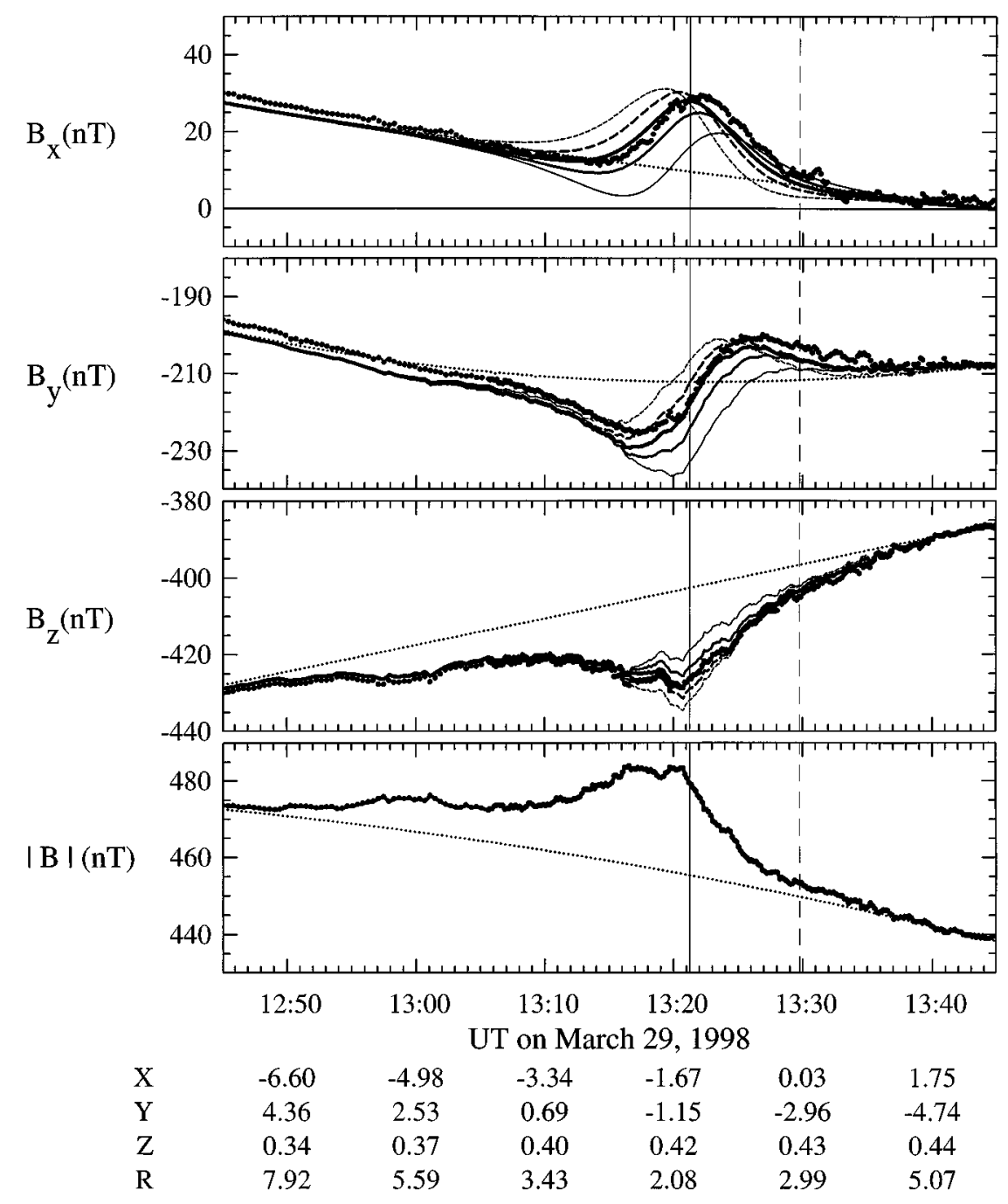

FIG. 6-Continued

The importance of the mantle for magnetic induction is less easily ruled out. Although dry rocks at room temperature and pressure are poor conductors, the presence of an aqueous phase or a significant increase in temperature can raise the conductivity by many orders of magnitude to above Europa and Callisto's reference conductivity $\sigma_{\mathrm{m}}$. In the case of the Earth, shallow-depth conductivities of the order of $100 \mathrm{mS} / \mathrm{m}$ appear to have been measured only under the oceans (Parkinson 1983, p. 327). At room temperatures, the conductivity of water-bearing rocks is determined by the conductivity of the electrolyte present in the pores and can again be approximated by Archie's law (see Section 5) with coefficients $C \sim 1$ and $m \sim 2$ (Keller and Frischknecht 1966). If the conductivity of the electrolyte $\sigma_{\text {liq }}$ is $\sim 3 \mathrm{~S} / \mathrm{m}$ (Section 5), then an aqueous volume fraction $q$ of order 15 and $8 \%$ is needed to produce a bulk conductivity of 60 and $20 \mathrm{mS} / \mathrm{m}$, respectively. A higher temperature could increase $\sigma_{\text {liq }}$ and thus reduce the amount of water required. However, to aug- ment $\sigma_{\text {liq }}$ by a factor 10 (and thus reduce the required $q$ by a factor $\sim 3$ ), the temperature must be increased from 18 to about $380^{\circ} \mathrm{C}$, at which point the dielectric properties of water and the conductivity of the fluid begin to break down (Keller and Frischknecht 1966, p. 31; Hermance 1995). Dry rocks can reach conductivities of several tens of millisterradians per meter or more, but only at temperatures of several hundreds of degrees Celsius where semiconduction or partial melt become significant (Hermance 1995). Given that the conducting material must be located no more than $\sim 200$ and $\sim 300 \mathrm{~km}$ below the surface of Europa and Callisto, respectively (see Section 5), such high temperatures are likely to force the overlying ice layer to melt, as was noted by Kuramoto et al. (1998), unless very large temperature gradients can be maintained. If the ice layer is melted to the point that it can carry the currents required by the observations (i. e., if it satisfies the conditions discussed in Section 5), then the underlying mantle is effectively shielded from the time-varying field and plays 
TABLE II

Constraints on the C onducting Layers of E uropa and C allisto

\begin{tabular}{|c|c|c|c|c|c|c|c|c|c|}
\hline \multirow[b]{2}{*}{$A$} & \multicolumn{5}{|c|}{ Europa } & \multicolumn{4}{|c|}{ Callisto } \\
\hline & $\begin{array}{l}\text { Max. } d \\
(\mathrm{~km})^{a}\end{array}$ & $\begin{array}{l}\text { Min. } \sigma \\
(\mathrm{mS} / \mathrm{m})^{b}\end{array}$ & $\begin{array}{l}\text { Min. } \sigma \text { for } \\
h_{\mathrm{c}}=100 \mathrm{~km} \\
(\mathrm{mS} / \mathrm{m})^{c}\end{array}$ & $\begin{array}{c}\text { Min. } \sigma \text { for } \\
h_{\mathrm{c}}=200 \mathrm{~km} \\
(\mathrm{mS} / \mathrm{m})^{c}\end{array}$ & $\begin{array}{c}\text { Min. } h \text { for } \\
\sigma=2.75 \mathrm{~S} / \mathrm{m} \\
(\mathrm{km})^{d}\end{array}$ & $\begin{array}{l}\text { Max. } d \\
(\mathrm{~km})^{a}\end{array}$ & $\begin{array}{l}\operatorname{Min} . \sigma \\
(\mathrm{mS} / \mathrm{m})^{b}\end{array}$ & $\begin{array}{c}\text { Min. } \sigma \text { for } \\
h_{\mathrm{c}}=350 \mathrm{~km} \\
(\mathrm{mS} / \mathrm{m})^{c}\end{array}$ & $\begin{array}{c}\text { Min. } h \text { for } \\
\sigma=2.75 \mathrm{~S} / \mathrm{m} \\
(\mathrm{km})^{d}\end{array}$ \\
\hline 0.3 & 516 & 10 & 22 & 20 & 1 & 796 & 4 & 7 & 0.6 \\
\hline 0.4 & 411 & 15 & 34 & 28 & 1.5 & 634 & 6 & 10 & 0.9 \\
\hline 0.5 & 322 & 23 & 65 & 38 & 2 & 497 & 9 & 13 & 1.1 \\
\hline 0.6 & 244 & 35 & 86 & 51 & 2.5 & 377 & 13 & 18 & 1.5 \\
\hline 0.7 & 175 & 58 & 116 & 72 & 3.5 & 270 & 22 & 26 & 2 \\
\hline 0.8 & 112 & 115 & 169 & 117 & 4.5 & 173 & 44 & 44 & 2.5 \\
\hline 0.9 & 54 & 344 & 348 & 344 & 7.5 & 83 & 131 & 323 & 4 \\
\hline
\end{tabular}

${ }^{a}$ Largest depth $d$ of the conducting shell (=thickness of the insulating outer crust) consistent with $A$ for an arbitrary shell conductivity $\sigma$.

${ }^{b}$ Smallest conductivity $\sigma$ consistent with $A$ for an arbitrary shell thickness $h$.

${ }^{c}$ Smallest conductivity $\sigma$ consistent with $A$ and a conducting shell not thicker than the $\mathrm{H}_{2} \mathrm{O}$ crust, of assumed thickness $h_{\mathrm{c}}$ (taken as 100 or 200 km for Europa, and $350 \mathrm{~km}$ for Callisto).

${ }^{d}$ Smallest shell thickness $h$ consistent with $A$, assuming the shell conductivity is $\sigma=2.75 \mathrm{~S} / \mathrm{m}$.

no role for induction, even if its conductivity is large. Also, we note that although mineralogical transitions are responsible for high conductivity regions in the Earth's lower mantle, the intense pressures required for these transitions cannot be attained in Europa and Callisto due to their much lower gravity. Finally, we point out that the above discussion did not take into account the depth of the silicate conductor. As mentioned in Section 5, a deeper conductor must be even more conducting to explain the observed field amplitude (e.g., for a depth of $100 \mathrm{~km}$ under Europa's surface, an amplitude $A=0.7$ requires a conductivity larger than $200 \mathrm{mS} / \mathrm{m}$; see Table III and the end of Section 3).

\subsection{Influence of an Ionosphere/Pick-up Cloud}

A conducting medium outside the moon could be provided by a collisional ionosphere or a cloud of pick-up ions. In either case, the conducting body would be larger than the moon itself $\left(r_{0}>r_{\mathrm{m}}\right)$. Consequently, the currents could be flowing closer to the spacecraft and therefore increase the magnitude of the inductive response for a fixed normalized conductivity. In addition, the reference conductivity $\sigma_{0}$ is inversely proportional to $r_{0}^{2}$ (see the end of Section 3 ) and therefore smaller for a larger conductor. This means that a given normalized conductivity $\sigma / \sigma_{0}$ corresponds to a smaller $\sigma$. As a result, one can obtain the same value of $A$ for a conductor of outer radius $r_{\mathrm{m}}$ and conductivity $\sigma_{1}$ or for a conductor of radius $r_{0}>r_{\mathrm{m}}$ and $\sigma<\sigma_{1}$. In Table III, we tabulated for different conductor sizes $r_{0}$ the conductivity $\sigma$ required to produce a signature with an amplitude $A>0.7$, along with the corresponding $\sigma_{0}$ and $A_{0}$ (defined in the last paragraph of Section 3). For a conductor reaching up to $690 \mathrm{~km}$ altitude above Europa (the distance of closest approach on flyby $\mathrm{E} 4)$, the requirement on the conductivity is only $\sigma>3.4 \mathrm{mS} / \mathrm{m}$.

TABLE III

R equirements on the C onductivity for C onductors of D ifferent Sizes

\begin{tabular}{|c|c|c|c|c|c|}
\hline Moon & $\begin{array}{l}\text { Outer radius of the } \\
\text { conducting shell, } r_{0}\end{array}$ & $\begin{array}{c}\text { Reference conductivity } \\
\sigma_{0} \text { for a conductor of } \\
\text { outer radius } r_{0}(\mathrm{mS} / \mathrm{m})^{a}\end{array}$ & $\begin{array}{l}\text { Inductive amplitude } A_{0} \\
\text { normalized to a perfect } \\
\text { conductor of radius } r_{0} \\
\text { required for } A>0.7^{b}\end{array}$ & $\begin{array}{l}\text { Normalized conductivity } \\
\sigma / \sigma_{0} \text { required for } A>0.7^{c}\end{array}$ & $\begin{array}{l}\text { Conductivity } \sigma \text { required } \\
\text { for } A>0.7(\mathrm{mS} / \mathrm{m})^{d}\end{array}$ \\
\hline \multirow[t]{4}{*}{ Europa } & $R_{E}-100 \mathrm{~km}$ & 4.8 & 0.85 & 44 & 211 \\
\hline & $R_{E}=1560 \mathrm{~km}$ & 4.2 & 0.7 & 14 & 58 \\
\hline & $R_{E}+300 \mathrm{~km}$ & 2.9 & 0.41 & 3.5 & 10 \\
\hline & $R_{E}+690 \mathrm{~km}$ & 2.0 & 0.23 & 1.7 & 3.4 \\
\hline \multirow[t]{2}{*}{ Callisto } & $R_{C}=2409 \mathrm{~km}$ & 1.6 & 0.7 & 14 & 22 \\
\hline & $R_{C}+421 \mathrm{~km}$ & 1.2 & 0.43 & 4.2 & 4.9 \\
\hline
\end{tabular}

\footnotetext{
${ }^{a}$ Given by $\sigma_{0}=\sigma_{\mathrm{m}}\left(r_{\mathrm{m}} / r_{0}\right)^{2}$ (see end of Section 3) with $\sigma_{\mathrm{m}}=4.2 \mathrm{mS} / \mathrm{m}$ and $r_{\mathrm{m}}=R_{E}$ for Europa, $\sigma_{\mathrm{m}}=1.6 \mathrm{mS} / \mathrm{m}$ and $r_{\mathrm{m}}=R_{\mathrm{C}}$ for Callisto.

${ }^{b}$ Given by $A_{0}=0.7\left(r_{\mathrm{m}} / r_{0}\right)^{3}$ (see end of Section 3 ).

${ }^{c}$ Obtained from $A_{0}$ and Fig. 2 (see end of Section 3). The requirement is valid for an arbitrary shell thickness.

${ }^{d}$ Obtained from $\sigma / \sigma_{0}$ and the reference conductivity $\sigma_{0}$.
} 
Similarly, for a conductor extending from Callisto's surface up to an altitude of $421 \mathrm{~km}$ (the closest approach distance for C9), the conductivity must only be $\sigma>4.9 \mathrm{mS} / \mathrm{m}$. These constraints are much weaker than those obtained in Section 5 for a conductor no larger than the moon itself.

In order to determine if such conductivities could be explained by an extended ionosphere, we now evaluate the Pedersen conductivity, which can be written

$$
\sigma_{\mathrm{P}}=\frac{n_{\mathrm{e}} e}{2 B}\left[\frac{2 \Omega_{\mathrm{i}} / v_{\mathrm{in}}}{1+\left(\Omega_{\mathrm{i}} / v_{\mathrm{in}}\right)^{2}}+\frac{2 \Omega_{\mathrm{e}} / v_{\mathrm{en}}}{1+\left(\Omega_{\mathrm{e}} / v_{\mathrm{en}}\right)^{2}}\right]
$$

(e.g., Hargreaves 1979, p. 85). Here, $n_{\mathrm{e}}$ is the electron density, $e$ the elementary charge, $\Omega_{\mathrm{e}}$ and $\Omega_{\mathrm{i}}$ the electron and ion gyrofrequencies, and $v_{\text {en }}$ and $v_{\text {in }}$ the electron-neutral and ion-neutral collision frequencies, and we assumed a single species of singly charged ions. Both terms inside the brackets reach their maximum of 1 only for $\Omega_{\mathrm{e}}=v_{\mathrm{en}}$ and $\Omega_{\mathrm{i}}=v_{\mathrm{in}}$. The collision frequencies $v_{\text {en }}=n_{\mathrm{n}} \hat{\sigma}_{\text {en }}\left(k T_{\mathrm{e}} / m_{\mathrm{e}}\right)^{1 / 2}$ and $v_{\text {in }}=n_{\mathrm{n}} \hat{\sigma}_{\text {in }}\left(k T_{\mathrm{i}} / m_{\mathrm{i}}\right)^{1 / 2}$ depend on the neutral density $n_{\mathrm{n}}$, the electron and ion masses $m_{\mathrm{e}}$ and $m_{\mathrm{i}}$, the electron-neutral and ion-neutral collision cross sections $\hat{\sigma}_{\text {en }}$ and $\hat{\sigma}_{\text {in }}$, and the electron and ion temperatures $T_{\mathrm{e}}$ and $T_{\mathrm{i}}(k$ is the Boltzmann constant). The cross section $\hat{\sigma}_{\text {in }}$ falls within the range $5-50 \times 10^{-19} \mathrm{~m}^{2}$ and $\hat{\sigma}_{\mathrm{en}} \approx 4 \times 10^{-19} \mathrm{~m}^{2}$ (Krall and Trivelpiece 1973, p. 321), and we take an ion mass $m_{\mathrm{i}}$ of $16 \mathrm{amu}$. Provided that the ion and electron temperatures are comparable, $\Omega_{\mathrm{e}} / v_{\text {en }}$ exceeds $\Omega_{\mathrm{i}} / v_{\text {in }}$ by a factor $\sim 200$. It follows that if the ion term inside the bracket becomes significant compared to 1 , then the electron term is negligible and vice versa, so that $\sigma_{\mathrm{P}}<n_{\mathrm{e}} e / 2 B$ to a good approximation. From six different Galileo radio occultation observations (two from encounter E4 and four from E6), Kliore et al. (1997) inferred an average electron density profile at Europa with a maximum at the surface of $1.1 \times 10^{10} \mathrm{~m}^{-3}$ and a scale height $>200 \mathrm{~km}$ below an altitude of $300 \mathrm{~km}$, and $>380 \mathrm{~km}$ above. Since $B>400 \mathrm{nT}$, the conductivity $\sigma_{\mathrm{P}}$ is everywhere $<2.2 \mathrm{mS} / \mathrm{m}$. Above $300 \mathrm{~km}$ altitude, the electron density is $<2.5 \times 10^{9} \mathrm{~m}^{-3}$ and thus $\sigma_{\mathrm{P}}<0.5 \mathrm{mS} / \mathrm{m}$, which even for a conductor extending up to $690 \mathrm{~km}$ above the surface is effectively insulating (compare to the $3.4 \mathrm{mS} / \mathrm{m}$ required for $A>0.7$ as mentioned above, and see Section 3). Hence it is appropriate to apply our single-shell model to the portion of the ionosphere between $r_{\mathrm{m}}$ and $r_{0}=r_{\mathrm{m}}+300 \mathrm{~km}$ and thereby neglect the conductivity at higher altitudes. For a conductor of this size to produce the $A>0.7$ signature, the required conductivity is $\sigma>10 \mathrm{mS} / \mathrm{m}$ (see Table III). This value exceeds by roughly a factor 5 the strong upper limit of the Pedersen conductivity computed above, thus allowing us to rule out any significant contribution to induction by ionospheric currents at Europa. For Callisto, no evidence for a current-carrying ionosphere has been reported yet and an estimation of its importance for induction must await the availability of relevant measurements or models.

In addition to ion-neutral collisions in the ionosphere, the separation of charges generated by the pick-up of fresh ions, e.g., by ionization of neutrals, in the vicinity of the moon produces a Pedersen conductivity $\dot{\rho} / B^{2}$ (e.g., Hill et al. 1983 , p. 371), where $\dot{\rho}$ is the mass loading rate, i.e., the net mass of new ions introduced in the environment per unit volume and time. If $n_{\mathrm{n}}$ is the number density of the neutrals being ionized, $m$ is the average mass and $\tau$ the average ionization lifetime of such a particle, then the mass loading rate can be written $\dot{\rho}=m n_{\mathrm{i}} / \tau$. At Europa, the source of new ions is electron impact ionization of the atmospheric $\mathrm{O}_{2}$ molecules (e.g., Saur et al. 1998). Using farultraviolet spectra of Europa acquired by the Hubble Space Telescope, Hall et al. (1998) estimated $\mathrm{O}_{2}$ column densities in the range of $2.4-14 \times 10^{18} \mathrm{~m}^{-2}$. Assuming a neutral atmospheric scale height of $150 \mathrm{~km}$ favored by the numerical simulation of Saur et al. (1998) (and not very different from the preferred $175 \mathrm{~km}$ of the MHD model by Kabin et al. 1999), this yields a maximum $\mathrm{O}_{2}$ density of about $10^{14} \mathrm{~m}^{-3}$. With an average $\mathrm{O}_{2}$ ionization time of $6.7 \times 10^{5} \mathrm{~s}$ (Ip 1996), we obtain $\dot{\rho}<5 \times 10^{9}$ amu $\mathrm{m}^{-3} \mathrm{~s}^{-1}$ and a pick-up Pedersen conductivity $<5 \times 10^{-2} \mathrm{mS} / \mathrm{m}$, almost two orders of magnitude less than the required value derived above, even if a cloud of this conductivity extended up to the altitude of the spacecraft during encounter E4. At Callisto, ions may be injected into the surrounding medium by photoionization of the thin $\mathrm{CO}_{2}$ atmosphere recently discovered with the Galileo near-infrared mapping spectrometer (Carlson 1999). To our knowledge, no other atmospheric constituents have been reported yet. With a maximum $\mathrm{CO}_{2}$ density at the surface $<4 \times 10^{14} \mathrm{~m}^{-3}$ and a lifetime against photoionization of approximately 2 years (Carlson 1999, Huebner et al. 1992), the local mass loading rate $\dot{\rho}$ is $<2.8 \times 10^{8} \mathrm{amu} \mathrm{m}^{-3} \mathrm{~s}^{-1}$, and with $B>30 \mathrm{nT}$, the pick-up conductivity is $<0.5 \mathrm{mS} / \mathrm{m}$, which is insufficient by an order of magnitude to account for the observations.

Thus for Europa and probably also for Callisto, the conductivities due to either ion pick-up or an ionosphere fail to explain the measured magnetic signature. This justifies the assumption, used in the remainder of this paper, that the conducting regions are confined to the interiors of the moons.

\section{CONCLUSION}

We have shown that the magnetic perturbations measured by Galileo during flybys of Europa and Callisto can to a large part be explained by invoking a dipole field induced by the time-varying magnetospheric background field of Jupiter. For Callisto, the perturbation cannot be explained by a permanent dipole field. For Europa, measurements from pass E26, while the moon was in the opposite magnetic hemisphere, have also definitely excluded a permanent dipole moment (Kivelson et al. 2000). The magnetic signatures at both moons are consistent with more than $70 \%$ of the induced dipole moment expected for perfectly conducting spheres the size of the moons. We showed that this requires currents flowing in a shell of high conductivity (at least about $60 \mathrm{mS} / \mathrm{m}$ for Europa and $20 \mathrm{mS} / \mathrm{m}$ for Callisto) reaching close to the surface of the moons (within 200-300 km 
depth) and examined various explanations for the required high conductivities. We showed that subsurface oceans with a salinity typical of the Earth's oceans and a few kilometers thickness can easily produce the observed induction response. We argued that solid ice, an ionosphere, or a cloud of pick-up ions are too resistive to explain the observations at Europa and probably also at Callisto, even if we take into account the potentially larger size of the two latter media. A conducting core, if any, is much too small to produce the amplitude of the observed field perturbation. Partial melt of the ice layer with a significant amount of brine inclusions or a hot silicate mantle could lower the resistivity sufficiently, but it seems unlikely that the required heat at relatively shallow depth would stop short of melting more extensive ocean-like regions in the ice crust that covers the mantle. The same holds for water mixed with rocks in the silicate mantle unless some interaction with the rocks can keep the electrolyte from freezing at temperatures much lower than those in the overlying ice crust. We thus confirm that the most likely explanation for the Galileo magnetometer observations is induction by subsurface oceans on Europa and Callisto. For Europa, our findings are in agreement with a similar analysis by Kuramoto et al., as well as surface observations that suggest a liquid ocean at shallow depth under a rigid ice crust. The magnetic evidence is of particular interest in so far as it indicates the existence of the ocean at the present epoch, not at some more or less recent past. The magnetic field observations are also the only evidence so far that such an ocean may exist on Callisto, whose surface does not provide any indication of an underlying liquid medium.

The difference between the observed field and our vacuum model of the perfect conductor response can plausibly be attributed to currents flowing in the ambient plasma, although no detailed modeling was attempted here. No evidence was found for an amplitude reduction of the induced field or a phase lag that would indicate an inductive response substantially weaker than that of a perfect conductor. However, the presence of plasma disturbances, uncertainties in the determination of the background field, and the limited coverage provided by simple flybys greatly limit the sensitivity of the Galileo observations to the characteristics of the induced fields. Detailed numerical modeling of the plasma interaction (e.g., Saur et al. 1998, Kabin et al. 1999) and the background field may help narrow our estimate of the induced fields and thus further constrain the conductivity distribution. Observational studies using Galileo data of the modification of Alfvén wings by induced fields, as predicted by Neubauer (1999), may also provide useful constraints. More extensive time and space coverage of the magnetic field in the vicinity of the moon, such as possible with an orbiting spacecraft, would clearly allow a much more detailed investigation of the conductivity distribution of the moons. Such an analysis could include the inductive response to the various nonsynodic frequencies of the background field and address more complicated electrical structures involving multiple shells and departures from spherical symmetry. It could thus provide a unique means of sounding the interior of the moons and the spatial extent of any subsurface oceans.

\section{APPENDIX: BESSEL FUNCTIONS}

The Bessel functions used in Eqs. (5) and (6) can be expressed as follows (see, e.g., Gradshteyn and Ryzhik 1979, p. 966, formulas 8.464):

$$
\begin{aligned}
J_{1 / 2}(z) & =\sqrt{\frac{2}{\pi z}} \sin z \\
J_{3 / 2}(z) & =\sqrt{\frac{2}{\pi z}}(\sin z / z-\cos z) \\
J_{5 / 2}(z) & =\sqrt{\frac{2}{\pi z}}\left[\left(3 / z^{2}-1\right) \sin z-3 \cos z / z\right] \\
J_{-1 / 2}(z) & =\sqrt{\frac{2}{\pi z}} \cos z \\
J_{-3 / 2}(z) & =\sqrt{\frac{2}{\pi z}}(-\sin z-\cos z 2 / z) \\
J_{-5 / 2}(z) & =\sqrt{\frac{2}{\pi z}}\left[\left(3 / z^{2}-1\right) \cos z+3 \sin z / z\right] .
\end{aligned}
$$

\section{ACKNOWLEDGMENTS}

The authors thank the referees William McKinnon and Konstantin Kabin, as well as Martin Volwerk and William Moore, for helpful comments about the manuscript, Joe Mafi for determining the background fields from Galileo measurements, and Richard Sadakane for help in realizing Fig. 2. This research was supported by the National Aeronautics and Space Administration under Contracts JPL 958694 and NAG 5-7959. This is UCLA Institute of Geophysics and Planetary Physics Publication 5422.

\section{REFERENCES}

Anderson, J. D., E. L. Lau, W. L. Sjogren, G. Schubert, and W. B. Moore 1998a. Europa's differentiated internal structure: Inferences from two Galileo encounters. Science 276, 1236-1239.

Anderson, J. D., G. Schubert, R. A. Jacobson, E. L. Lau, W. B. Moore, and W. L. Sjogren 1998b. Distribution of rock, metals, and ices in Callisto. Science 280, 1573-1576.

Carlson, R. W. 1999. A tenuous carbon dioxide atmosphere on Jupiter's moon Callisto. Science 283, 820-821.

Carr, M. H., M. J. S. Belton, C. R. Chapman, M. E. Davies, P. Geissler, R. Greenberg, A. S. McEwen, B. R. Tufts, R. Greeley, R. Sullivan, J. W. Head, R. T. Pappalardo, K. P. Klaasen, T. V. Johnson, J. Kaufman, D. Senske, J. Moore, G. Neukum, G. Schubert, J. A. Burns, P. Thomas and J. Veverka 1998. Evidence for a subsurface ocean on Europa. Nature 391, 363-365.

Colburn, D. S., and R. T. Reynolds 1985. Electrolytic currents in Europa. Icarus 63, 39-44.

Cole, G. H. A. 1994. The physics of planetary interiors. Rep. Prog. Phys. 57, 755-820.

Connerney, J. E. P. 1992. Doing more with Jupiter's magnetic field. In Planetary Radio Emissions III (H. O. Rucker, S. J. Bauer, and M. L. Kaiser, Eds.), p. 13. Oesterreichische Akad. der Wiss., Vienna.

Gradshteyn, I. S., and I. M. Ryzhik 1979. Table of Integrals, Series, and Products, p. 966. Academic Press, London.

Hall, D. T., P. D. Feldman, M. A. McGrath, and D. F. Strobel 1998. The far-ultraviolet oxygen airglow of Europa and Ganymede. Astrophys. J. 499, 475-481.

Hargreaves, J. K. 1979. The Upper Atmosphere and Solar-Terrestrial Relations, p. 85. Van Nostrand Reinhold, Wokingham, England. 
Hermance, J. F. 1995. Electrical conductivity models of the crust and mantle. In Global Earth Physics: A Handbook of Physical Constants (T. J. Ahrens, Ed.), pp. 190-205. American Geophysical Union, Washington, DC.

Hill, T. W., A. J. Dessler, and C. K. Goertz 1983. Magnetospheric models. In Physics of the Jovian Magnetosphere (A. J. Dessler, Ed.), pp. 353-394. Cambridge Univ. Press, New York.

Hoppa, G. V., B. R. Tufts, R. Greenberg, and P. E. Geissler 1999. Formation of cycloidal features on Europa. Science 285, 1899-1902.

Huebner, W. F., J. J. Keady, and S. P. Lyon 1992, Solar photo rates for planetary atmospheres and atmospheric pollutants. Astrophys. Space Sci. 195, 1-294.

Ip, W.-H. 1996. Europa's oxygen exosphere and its magnetospheric interaction. Icarus 120, 317-325.

Kabin, K., M. R. Combi, T. I. Gombosi, A. F. Nagy, D. L. DeZeeuw, and K. G. Powell 1999. On Europa's magnetospheric interaction: A MHD simulation of the E4 flyby. J. Geophys. Res. 104, 19,983-19,992.

Kargel, J. S., and G. J. Consolmagno 1996. Magnetic fields and the detectability of brine oceans in Jupiter's icy satellites. Lunar. Planet. Sci. 27, 643-644.

Keller, G. V., and F. C. Frischknecht 1966. Electrical Methods in Geophysical Prospecting, pp. 1-60. Pergamon Press, Oxford.

Khurana, K. K. 1997. Euler potential models of Jupiter's magnetospheric field, J. Geophys. Res. 102, 11,295-11,306.

Khurana, K. K., M. G. Kivelson, D. J. Stevenson, G. Schubert, C. T. Russell, R. J. Walker, and C. Polanskey 1998. Induced magnetic fields as evidence for subsurface oceans in Europa and Callisto. Nature 395, 777-780.

Kivelson, M. G., K. K. Khurana, C. T. Russell, M. Volwerk, R. J. Walker, and C. Zimmer 2000. Galileo magnetometer measurements strengthen the case for a subsurface ocean at Europa. Science, in press.

Kivelson, M. G., K. K. Khurana, D. J. Stevenson, L. Bennett, S. Joy, C. T. Russell, R. J. Walker, C. Zimmer, and C. Polanskey 1999. Europa and Callisto: Induced or intrinsic fields in a periodically varying plasma environment. J. Geophys. Res. 104, 4609-4625.

Kliore, A. J., D. P. Hinson, F. M. Flasar, A. F. Nagy, and T. E. Cravens 1997. The ionosphere of Europa from Galileo radio occultations. Science 277, 355-358.

Krall, N. A., and A. W. Trivelpiece 1973. Principles of Plasma Physics. McGraw-Hill, New York.
Kuramoto, K., Y. Saiganji, and T. Yamamoto 1998. Oscillating magnetic dipole moment of Europa induced by jovian magnetic field: A probe for detecting Europa's ocean. Lunar. Planet. Sci. 29, 1254.

Montgomery, R. B. 1963. Oceanographic data. In American Institute of Physics Handbook (D. E. Gray, Ed.), pp. 125-127. McGraw-Hill, New York.

Neubauer, F. M. 1998. The sub-Alfvénic interaction of the Galilean satellites with the jovian magnetosphere. J. Geophys. Res. 103, 19,84319,866 .

Neubauer, F. M. 1999. Alfvén wings and electromagnetic induction in the interiors: Europa and Callisto. J. Geophys. Res. 104, 28,671-28,684.

Olhoeft, G. R. 1989. Electrical properties of rocks. In Cindal Data Series on Material Properties (C. Y. Ho, Ed.), Vol. II-2, pp. 257-330. Hemisphere Publishing, New York.

Pappalardo, R. T., J. W. Head, R. Greeley, R. J. Sullivan, C. Pilcher, G. Schubert, W. B. Moore, M. H. Carr, J. M. Moore, M. J. S. Belton, and D. L. Goldsby 1998. Geological evidence for solid-state convection in Europa's ice shell. Nature 391, 365-368.

Parkinson, W. D. 1983. Introduction to Geomagnetism, pp. 308-340. Scottish Academic Press, Edinburgh.

Rathbun, J. A., G. S. Musser, and S. W. Squyres 1998. Ice diapirs on Europa: Implications for liquid water. Geophys. Res. Lett. 25, 4157-4160.

Rikitake, T. 1966. Electromagnetism and the Earth's Interior, pp. 128-134. Elsevier, Amsterdam.

Saur, J., D. F. Strobel, and F. M. Neubauer 1998. Interaction of the jovian magnetosphere with Europa: Constraints on the neutral atmosphere. J. Geophys. Res. 103, 19,947-19,962.

Squyres, S. W., R. T. Reynolds, P. M. Cassen, and S. J. Peale 1983. Liquid water and active resurfacing on Europa. Nature 301, 225-226.

Stacey, F. D. 1992. Physics of the Earth, pp. 363-368. Brookfield Press, Brisbane.

Vasyliunas, V. 1983. Plasma distribution and flow. In Physics of the Jovian Magnetosphere (A. J. Dessler, Ed.), pp. 395-453. Cambridge Univ. Press, New York.

Watanabe, T., and K. Kurita 1993. The relationship between electrical conductivity and melt fraction in a partially molten simple system: Archie's law behavior. Phys. Earth Planet. Inter. 78, 9-17. 